\title{
LOCAL LIMIT THEOREMS FOR TRANSITION DENSITIES OF MARKOV CHAINS CONVERGING TO DIFFUSIONS *
}

\author{
Valentin KONAKOV \\ Central Economics Mathematical Institute, Academy of Sciences \\ Nahimovskii av. 47, 117418 Moscow, Russia \\ Enno MAMMEN \\ Institut für Angewandte Mathematik, Ruprecht-Karls-Universität Heidelberg \\ Im Neuenheimer Feld 294, 69120 Heidelberg, Germany \\ E mail: mammen@statlab.uni-heidelberg.de \\ FAX ++496221 5331
}

September 15, 1998

We consider triangular arrays of Markov chains that converge weakly to a diffusion process. Local limit theorems for transition densities are proved.

1991 MSC: primary $62 \mathrm{G} 07$, secondary $60 \mathrm{G} 60$

Keywords and phrases: Markov chains, diffusion processes, transition densities

Short title: Local limit theorems of Markov chains.

*This research was supported by grant 436 - RUS $113 / 467 / 1$ from the Deutsche Forschungsgemeinschaft and by grants 98-01-04108 and 98-01-00524 from the Russian Foundation of Fundamental Researches. 


\section{Introduction.}

In this paper we consider triangular arrays of Markov chains that converge weakly to a diffusion process. Our main result is that transition densities converges with parametric rate $\left[\right.$ i.e. $\left.\mathrm{O}\left(n^{-1 / 2}\right)\right]$ to the transition density in the diffusion model.

This research is motivated by recent new approaches in time series analysis. In a series of papers [see e.g. Doukhan and Nze (1995), Franke, Kreiss and Mammen (1996), Masry and Tjøstheim (1994), Robinson (1983), Tjøstheim (1994), Tjøstheim and Auestad (1994)] it has been proposed to use nonparametric approaches to model time series. In particular nonparametric autoregression models have been considered:

$$
X(k+1)=m(X(k))+\sigma(X(k)) \varepsilon(k+1),
$$

where the innovations $\varepsilon(1), \varepsilon(2), \ldots$ are typically assumed to be i.i.d. mean zero variables. For the functions $m$ and $\sigma$ nonparametric smoothness assumptions are made and nonparametric smoothing methods are proposed for their estimation. For a discussion of different nonparametric statistical problems in these models we refer to the references above. Under regularity conditions on $m, \sigma$ and the distribution of $\varepsilon(i)$, solutions of (1.1) are stationary processes. In Dahlhaus (1997) models are proposed for time series that are not stationary, however locally stationary. In particular he considers autoregressive processes with time varying coefficients:

$$
X_{n}(k+1)=a\left(\frac{k}{n}\right) X_{n}(k)+\varepsilon(k+1) .
$$

In this paper we discuss another model and use as in (1.2) the asymptotic approach that we observe a function $a$ on a finer grid for $n \rightarrow \infty$. We consider the model

$$
X_{n}(k+1)=X_{n}(k)+\frac{1}{n} m\left\{\frac{k}{n}, X_{n}(k)\right\}+\frac{1}{\sqrt{n}} \varepsilon_{n}(k+1) .
$$

We make the Markov assumption that the conditional distribution of the innovation $\varepsilon_{n}(k+1)$ given the past $X_{n}(k), X_{n}(k-1), \ldots$ depends only on the last value $X_{n}(k)$. [For a slightly more general model see the next section.] It is well known that [under regularity conditions] the process $Y_{n}(s)=X_{n}\left(\kappa_{n}(s)\right)$ [where $\kappa_{n}(t)=\max \{k \leq n t\}$ ] converges to a diffusion process [see e.g. Skorohod (1987)]. The main result of this paper is that the conditional density of $Y_{n}(1)$ given $Y_{n}(0)$ converges with parametric rate $\left[\right.$ i.e. $\left.\mathrm{O}\left(n^{-1 / 2}\right)\right]$ to the conditonal density of the diffusion.

In particular, this result may be applied to discuss statistical nonparametric estimation problems of the transition density and the shift function $m$ under different smoothness and structural assumptions. Our result reduces the discussion of some of such problems in model (1.3) to the analysis of corresponding problems in diffusion models. For the discussion of some nonparametric estimation problems in diffusion models see Kutoyants $(1997,1998)$. In this paper we will not address statistical problems. 


\section{Results.}

For each $n \geq 1$ we consider Markov chains $X_{n}(k)$ where the time $k$ runs from 0 to $n$. The Markov chain $X_{n}$ is assumed to take values in $\mathbb{R}^{p}$. The dynamics of the chain $X_{n}$ is described by

$$
X_{n}(k+1)=X_{n}(k)+\Delta_{n}(k+1) m\left\{s_{n}(k), X_{n}(k)\right\}+\Delta_{n}(k+1)^{1 / 2} \varepsilon_{n}(k+1) .
$$

Here $\Delta_{n}(k)>0$ are real numbers with

$$
\sum_{k=1}^{n} \Delta_{n}(k)=1 .
$$

The numbers $s_{n}(k)$ are defined as $s_{n}(0)=0$ and

$$
s_{n}(k)=\sum_{i=1}^{k} \Delta_{n}(i) \quad \text { for } k \geq 1 \text {. }
$$

Furthermore, $m$ is a function $m:[0,1] \times \mathbb{R}^{p} \rightarrow \mathbb{R}^{p}$. The stochastic structure of the $\mathbb{R}^{p}$ valued error variables $\varepsilon_{n}(k)$ is described as follows. Given $X_{n}(i)=x(i)$ for $i=0, \ldots, k$ the variable $\varepsilon_{n}(k+1)$ has a conditional density $q\left\{s_{n}(k), x(k), \bullet\right\}$. The conditional covariance matrix of $\varepsilon_{n}(k+1)$ is denoted by $\Sigma\left\{s_{n}(k), x(k)\right\}$. Here $q$ is a function mapping $[0,1] \times \mathbb{R}^{p} \times \mathbb{R}^{p}$ into $\mathbb{R}_{+}$. Furthermore, $\Sigma$ is a function mapping $[0,1] \times \mathbb{R}^{p}$ into the set of positive definite $p \times p$ matrices. The Markov chain is supposed to start in a deterministic point $X_{n}(0)=x$. The conditional density of $X_{n}(n)$, given $X_{n}(0)=x$, is denoted by $p_{n}(x, \bullet)$. Study of the transition densities $p_{n}(x, z)$ is the topic of this paper. Conditions on $\Delta_{n}(k), m, q\left\{s_{n}(k), x(k), \bullet\right\}$ and $\Sigma\left\{s_{n}(k), x(k)\right\}$ will be given below.

By time change the Markov chain $X_{n}$ defines a process $Y_{n}$ on [0,1]. More precisely, put $\kappa_{n}(t)=\sup \left\{k: s_{n}(k) \leq t, 1 \leq k \leq n\right\}$. This defines a monotone time transform $\kappa_{n}:[0,1] \rightarrow\{1, \ldots, n\}$. Using this time transform we get the following process:

$$
Y_{n}(t)=X_{n}\left\{\kappa_{n}(t)\right\}
$$

Under our assumptions, see Section 3, the process $Y_{n}$ converges to a diffusion $Y(t)$. This follows for instance from Theorem 1, p. 82 in Skorohod (1987). The diffusion is defined by $Y(0)=x$ and

$$
d Y(t)=m\{t, Y(t)\} d t+\Lambda\{t, Y(t)\} d W(t),
$$

where $W$ is a $p$ dimensional Brownian motion. The matrix $\Lambda(t, z)$ is the unique symmetric matrix defined by $\Lambda(t, z) \Lambda(t, z)^{T}=\Sigma(t, z)$. The conditional density of $Y(1)$, given $Y(0)=x$, is denoted by $p(x, \bullet)$. Note that the conditional density of $Y_{n}(1)$, given $Y_{n}(0)=x$, is denoted by $p_{n}(x, \bullet)$. The following theorem contains our main result. It gives a bound for the rate of convergence of $p_{n}$ to $p$. 
Theorem 2.1 Assume (A1) - (A5). Then the following estimate holds:

$$
\sup _{x, y \in R^{p}}\left(1+\|y-x\|^{2\left(S^{\prime}-1\right)}\right)\left|p_{n}(x, y)-p(x, y)\right|=O\left(n^{-1 / 2}\right),
$$

where $S^{\prime}$ is defined in Assumption (A2). The norm $\|\ldots\|$ is the usual Euclidean norm.

Kasymdzganova (1981) considered the case of a homogeneous random walk on the lattice $\mathbb{Z}^{p}$ [with no drift, e.g. $m \equiv 0$ ]. She assumed the following conditional distribution for the innovations

$$
P\left(\varepsilon_{n}(k+1)= \pm e_{i} \mid \sqrt{n} X_{n}(k)=x\right)=\frac{1}{2 p}\left(1-\frac{1}{n} Q(x)\right),
$$

where $e_{1}=(1,0, \ldots, 0), \ldots, e_{p}=(0, \ldots, 1), x \in \mathbb{Z}^{p}$ and $\frac{1}{n} Q(x)$ is a probability of vanishing of a particle at $x$. For this scheme she proved that for $x \in \mathbb{Z}^{p}, y \in \mathbb{Z}^{p}$

$$
\lim _{n \rightarrow \infty} n^{p / 2} P\left(X_{n}(n)=y / \sqrt{n} \mid X_{n}(0)=x / \sqrt{n}\right)=p(t, x, y)
$$

where $p(t, x, y)$ is the fundamental solution of the equation

$$
\frac{\partial p(t, x, y)}{\partial t}=\frac{1}{2} \nabla_{x} p(t, x, y)-Q(x) p(t, x, y)
$$

with $p(0, x, y)=\delta(x-y)$ where $\delta$ is the Dirac function. Local limit theorems for homogeneous Markov chains with continuous state space and equidistant partitions were given in Konakov and Molchanov (1984).

We use the parametrix method. This approach is well known in the theory of partial differential equations [see Il'in, Kalashnikov and Oleinik (1962) and McKean and Singer (1967)] and was used e.g. in Kuznetsov (1998) to obtain bounds for Poisson kernels. But as far as we know for Markov chains the parametrix method was not systematically developed before.

\section{Conditions.}

For $t \in[0,1]$ and $x \in \mathbb{R}^{p}$ let $q\{t, x, \bullet\}$ be a density in $\mathbb{R}^{p}$. We make the following assumptions.

(A1)

$$
\begin{gathered}
\int q\{t, x, u\} u d u=0 \text { for all } t \in[0,1], x \in \mathbb{R}^{p}, \\
\int q\{t, x, u\} u_{i} u_{j} d u=\sigma_{i j}(t, x) \text { for all } t \in[0,1], x \in \mathbb{R}^{p} \text { and } i, j=1, \ldots, p .
\end{gathered}
$$

The matrix with elements $\sigma_{i j}(t, x)$ is denoted by $\Sigma(t, x)$. 
(A2) There exist a positive integer $S^{\prime}$ and a function $\psi: \mathbb{R}^{p} \rightarrow \mathbb{R}$ with $\sup _{x \in \mathbb{R}^{p}}|\psi(x)|<$ $\infty$ and $\int_{\mathbb{R}^{p}}\|x\|^{S}|\psi(x)| d x<\infty$ for $S=2 p S^{\prime}+4$ such that

$$
\begin{gathered}
\left|\mathrm{D}_{u}^{\nu} q\{t, x, u\}\right| \leq \psi(u) \quad \text { for all } t \in[0,1], x, u \in \mathbb{R}^{p}, \text { and }|\nu|=0, \ldots, 4, \\
\left|\mathrm{D}_{x}^{\nu} q\{t, x, u\}\right| \leq \psi(u) \text { for all } t \in[0,1], x, u \in \mathbb{R}^{p} \text { and }|\nu|=0, \ldots, 2 .
\end{gathered}
$$

[For the case that $S^{\prime}=1$ Theorem 2.1 can be shown under the weaker assumption that (A2) holds for a function $\psi$ with $\sup _{x \in \mathbb{R}^{p}}|\psi(x)|<\infty$ and $\int_{\mathbb{R}^{p}}\|x\|^{k}|\psi(x)| d x$ $<\infty$ for an integer $k>p+4$.]

(A3) There exist positive constants $c$ and $C$ such that

$$
c \leq\langle\Sigma(t, x) \theta, \theta\rangle \leq C
$$

for all $\theta,\|\theta\|=1, t$ and $x$.

(A4) There exists a constant $B$ with

$$
B^{-1}<\frac{\Delta_{n}(k)}{\Delta_{n}(l)}<B
$$

for $n \geq 1$ and $1 \leq k, l \leq n$. [Then it follows that $\Delta_{\max }=\max _{1 \leq j \leq n} \Delta_{n}(j)=$ $\left.O\left(n^{-1 / 2}\right) \cdot\right]$

(A5) The functions $m(t, x)$ and $\Sigma(t, x)$ and their first derivatives with respect to $x$ and with respect to $t$ are continuous and bounded (uniformly in $t$ and $x$ ). All these functions are Lipschitz continuous with respect to $x$ (with a Lipschitz constant that does not depend on $t)$. Furthermore, $\partial^{2} /\left(\partial x_{j} \partial x_{k}\right) \Sigma(t, x)$ exists for $1 \leq$ $j, k \leq p$ and is Holder continuous with respect to $x$ (with positive exponent $\delta$ and constant that do not depend on $t$ ).

\section{Examples and extensions.}

(i) The result can be extended to the case that $q, m$ and $\Sigma$ depend on $n$. For this purpose conditions (A2),.., (A5) have to be replaced by assumptions that hold uniformly in $n$.

(ii) Unbounded drift function. Our result can be extended to the case of an unbounded drift function $m$ that is of the form $b(t) x+a(t, x)$ where $a$ fullfills the conditions stated for $m$ and where $b(t)$ is a matrix that depends continuously on $t$.

(iii) Unbounded one step transition density. Our results can be extended to unbounded transition densities if the transition density for a finite number of steps is bounded, see e.g. (vii). 
(iv) Functionals of Markov chains. Our theorem implies that the density of $\left(Y_{n}\left(t_{1}\right)\right.$, $\left.\ldots, Y_{n}\left(t_{k}\right)\right)$ converges to the density of $\left(Y\left(t_{1}\right), \ldots, Y\left(t_{k}\right)\right)$ in $\mathrm{L}_{1}$ norm for any tuple $0 \leq t_{1}<\ldots<t_{k} \leq 1$. We conjecture that with the approach of Davydov (1980, 1981) these results can be used to show that the density of $H\left(Y_{n}(\bullet)\right)$ converges to the density of $H(Y(\bullet))$ for a wide range of functionals $H$.

(v) Conditional Markov chains. In particular, our result can be used to show that the conditional density of $\left(Y_{n}\left(t_{1}\right), \ldots, Y_{n}\left(t_{k}\right)\right)$ given $\left.Y_{n}(1)\right)$ converges to the conditional density of $\left(Y\left(t_{1}\right), \ldots, Y\left(t_{k}\right)\right)$ given $\left.Y(1)\right)$ (in $\mathrm{L}_{1}$ norm), where tuple $t_{1}, \ldots, t_{k}$ is a tuple with $0 \leq t_{1}<\ldots<t_{k}<1$.

(vi) Euler approximations. The case where $q$ is a normal density corresponds to Euler approximations that are the simplest strong Taylor approximations used as numerical solutions to stochastic differential equations, see Kloeden and Platen (1992).

(vii) Transport processes. Let us consider a symmetric and positively definite $p \times p$ matrix $S(x)$ and vector $m(x)=\left(m_{1}(x), \ldots, m_{p}(x)\right)^{T}$ where $x \in \mathbb{R}^{p}$. For $a>0$ we consider independent variables $R_{a, 1}, R_{a, 2}, \ldots, U_{a, 1}, U_{a, 2}, \ldots$ where $R_{a, i}$ have density $a^{-1} \exp (-r / a)$ and where $U_{a, i}$ are uniformly distributed on the unit sphere in $\mathbb{R}^{p}$. We define the following chain (transport processes, see e.g. Pinsky, 1991):

$$
\begin{aligned}
& X_{a}(i+1)=x, \\
& X_{a}(i+1)=X_{a}(i)+a^{2} m\left(X_{a}(i)\right)+S\left(X_{a}(i)\right) U_{a, i} R_{a, i}, \quad \text { for } 0 \leq i \leq\left[1 / a^{2}\right] .
\end{aligned}
$$

This process has no bounded one step transition density and it does not fulfill the conditions of our theorem for this reason. However it is easy to show that for a finite numbers $k$ of steps the transition density of $X_{a}(i+k)$ given $X_{a}(i)$ is bounded, so that we can apply our theorem to the process $i \rightarrow X_{a}(i k)$. This shows that the density $X_{a}\left(\left[1 / a^{2}\right]\right)$ converges to the density of the diffusion $Y$ at time point $t=1$ for $a \rightarrow 0$ where

$$
\begin{aligned}
Y(0) & =x, \\
d Y(t) & =c S(Y(t)) d W(t)+m(Y(t)) d t, \quad \text { for } 0 \leq t \leq 1,
\end{aligned}
$$

where $c$ is an appropriate constant. The speed of convergence is of order $O(a)$.

(viii) Lattice distributions. Our approach can be extended to obtain local limit theorems for a general class of nonhomogeneous random walks on a lattice $\mathbb{Z}^{p}$. An essential tool are finite difference methods for uniformly parabolic equations (see e.g. Thomée, 1990). This would generalize the results of Konovalov (1981) and Kasymdzganova (1981). 


\section{Proofs.}

For all $0 \leq j \leq n$ and $u, v \in \mathbb{R}^{p}$ we define additional Markov chains $\tilde{X}_{n}=\tilde{X}_{n, j, u, v}$. For fixed $j, u$ and $v$, the chain is defined for $i$ with $j \leq i \leq n$. The dynamics of the chain is described by

$$
\tilde{X}_{n}(j)=u
$$

and

$$
\tilde{X}_{n}(i+1)=\tilde{X}_{n}(i)+\Delta_{n}(i+1) m\left\{s_{n}(i), v\right\}+\Delta_{n}(i+1)^{1 / 2} \tilde{\varepsilon}_{n}(i+1) .
$$

The stochastic structure of the $\mathbb{R}^{p}$ valued error variables $\tilde{\varepsilon}_{n}(i)$ is described as follows. Given $\tilde{X}_{n}(l)=x(l)$ for $l=j, \ldots, i$ the variable $\tilde{\varepsilon}_{n}(i+1)$ has a conditional density $q\left\{s_{n}(i), v, \bullet\right\}$. Note that the conditional distribution of $\tilde{X}_{n}(i+1)-\tilde{X}_{n}(i)$ does not depend on the past $\tilde{X}_{n}(l)$ for $l=j, \ldots, i$. Let us call $\tilde{X}_{n}$ the Markov chain frozen at $v$. We put $\tilde{Y}_{n}(t)=\tilde{X}_{n}\left\{\kappa_{n}(t)\right\}$ and we write $\tilde{p}_{n}\left(s_{n}(j), s_{n}(k), u, v\right)$ for the conditional density of $\tilde{X}_{n}(k)\left[=\tilde{X}_{n, j, u, v}(k)\right]$ at the point $v$, given $\tilde{X}_{n}(j)=u$. Note that the variable $v$ acts here twice: as the argument of the density and as a defining quantity of the process $\tilde{X}_{n}=\tilde{X}_{n, j, u, v}$. Furthermore, we denote by $\tilde{p}_{n, j}^{v}(u, w)$ the conditional density of $\tilde{X}_{n}(j+1)\left[=\tilde{X}_{n, j, u, v}(j+1)\right]$ at the point $w$, given $\tilde{X}_{n}(j)=u$.

Similarly for $0<s<1$ and $u, v \in \mathbb{R}^{p}$ we define diffusions $\tilde{Y}=\tilde{Y}_{s, u, v}$ that are defined for $s \leq z \leq 1$ by

$$
\tilde{Y}(s)=u
$$

and

$$
d \tilde{Y}(z)=m\{z, v\} d z+\Lambda\{z, v\} d W(z) .
$$

Now $\tilde{p}(s, t, u, v)$ denotes the conditional density of $\tilde{Y}(t)\left[=\tilde{Y}_{s, u, v}(t)\right]$ at the point $v$, given $\tilde{Y}(s)=u$. Note again that the variable $v$ acts here twice: as the argument of the density and as a defining quantity of the process $\tilde{Y}=\tilde{Y}_{s, u, v}$. Furthermore, we denote by $\tilde{p}_{j}^{v}(u, w)$ the conditional density of $\tilde{Y}\left(s_{n}(j+1)\right)\left[=\tilde{Y}_{s_{n}(j), u, v}\left(s_{n}(j+1)\right)\right]$ at the point $w$, given $\tilde{Y}\left(s_{n}(j)\right)=u$. By definition, we have that

$$
\begin{aligned}
\tilde{p}(s, t, x, y)= & (2 \pi)^{-p / 2}\left(\operatorname{det} C_{y}(s, t)\right)^{-1 / 2} \\
& \exp \left[-\frac{1}{2}\left\{y-x-\gamma_{y}(s, t)\right\}^{\prime} C_{y}(s, t)^{-1}\left\{y-x-\gamma_{y}(s, t)\right\}\right],
\end{aligned}
$$

where

$$
\begin{aligned}
C_{y}(s, t) & =\int_{s}^{t} \Sigma(u, y) d u \\
\gamma_{y}(s, t) & =\int_{s}^{t} m(u, y) d u .
\end{aligned}
$$

Let us introduce the following infinitesimal operators $A_{n, j}$ and $\tilde{A}_{n, j}^{v}$ acting on functions $f: \mathbb{R}^{p} \rightarrow \mathbb{R}:$

$$
A_{n, j} f(u)=\frac{\int p_{n, j}(u, w) f(w) d w-f(u)}{\Delta_{n}(j+1)}
$$


and

$$
\tilde{A}_{n, j}^{v} f(u)=\frac{\int \tilde{p}_{n, j}^{v}(u, w) f(w) d w-f(u)}{\Delta_{n}(j+1)} .
$$

Here we write $p_{n, j}(u, \bullet)$ for $p_{n}\left(s_{n}(j), s_{n}(j+1), u, \bullet\right)$ where $p_{n}(s, t, u, \bullet)$ denotes the conditional density of $Y_{n}(t)$, given $Y_{n}(s)=u$. For $k>j$ we put

$$
H_{n}\left(s_{n}(j), s_{n}(k), u, v\right)=\left\{A_{n, j}-\tilde{A}_{n, j}^{v}\right\} f(u),
$$

where $f(u)=\tilde{p}_{n}\left(s_{n}(j+1), s_{n}(k), u, v\right)$. In the following we use the following convolution type binary operation $\otimes_{n}$ :

$\left(g \otimes_{n} f\right)\left(s_{n}(j), s_{n}(k), u, v\right)=\sum_{i=j}^{k-1} \Delta_{n}(i+1) \int_{\mathbb{R}^{p}} g\left(s_{n}(j), s_{n}(i), u, w\right) f\left(s_{n}(i), s_{n}(k), w, v\right) d w$,

where $0 \leq j<k \leq n$. In this definition the convention is used that $\sum_{i=j}^{k-1} \ldots=0$ if $j \geq k$. We write $g \otimes_{n} H_{n}^{(0)}$ for $g$ and for $r=1,2, \ldots, n$, we denote the $r$ fold "convolution" $\left(g \otimes_{n} H_{n}^{(r-1)}\right) \otimes_{n} H_{n}$ by $g \otimes_{n} H_{n}^{(r)}$. Our first lemma states a simple relation between $p_{n}$ and $\tilde{p}_{n}$ :

Lemma 5.1 For $0 \leq j<k \leq n$ the following formula holds:

$$
p_{n}\left(s_{n}(j), s_{n}(k), u, v\right)=\sum_{r=0}^{k-j}\left(\tilde{p}_{n} \otimes_{n} H_{n}^{(r)}\right)\left(s_{n}(j), s_{n}(k), u, v\right),
$$

where in the calculation of $\tilde{p}_{n} \otimes_{n} H_{n}^{(r)}$ we define

$$
p_{n}\left(s_{n}(j), s_{n}(j), x, y\right)=\tilde{p}_{n}\left(s_{n}(k), s_{n}(k), x, y\right)=\delta(x-y) .
$$

Here $\delta$ denotes the Dirac function.

Proof of Lemma 5.1. Note that by definition:

$$
\begin{aligned}
& H_{n}\left(s_{n}(j), s_{n}(k), u, v\right) \\
& \quad=\frac{\int\left[p_{n, j}(u, w)-\tilde{p}_{n, j}^{v}(u, w)\right] \tilde{p}_{n}\left(s_{n}(j+1), s_{n}(k), w, v\right) d w}{\Delta_{n}(j+1)} .
\end{aligned}
$$

Using the Markov property we get the following identity:

$$
\begin{aligned}
& p_{n}\left(s_{n}(j), s_{n}(k), u, v\right)-\tilde{p}_{n}\left(s_{n}(j), s_{n}(k), u, v\right) \\
&=\sum_{i=j}^{k-1} \Delta_{n}(i+1) \int p_{n}\left(s_{n}(j), s_{n}(i), u, w\right) \\
& \quad \frac{\left[p_{n, i}\left(w, w^{\prime}\right)-\tilde{p}_{n, i}^{v}\left(w, w^{\prime}\right)\right] \tilde{p}_{n}\left(s_{n}(i+1), s_{n}(k), w^{\prime}, v\right)}{\Delta_{n}(i+1)} d w^{\prime} d w \\
&= \sum_{i=j}^{k-1} \Delta_{n}(i+1) \int p_{n}\left(s_{n}(j), s_{n}(i), u, w\right) H_{n}\left(s_{n}(i), s_{n}(k), w, v\right) d w \\
&=\left(p_{n} \otimes_{n} H_{n}\right)\left(s_{n}(j), s_{n}(k), u, v\right) .
\end{aligned}
$$


The lemma follows by iterative application of this identity.

Let us introduce the following differential operators $L_{s}$ and $\tilde{L}_{s}^{y}$ :

$$
L_{s} f(u)=f^{\prime}(u)^{T} m(s, u)+\frac{1}{2} \operatorname{tr}\left[\Lambda(s, u)^{T} f^{\prime \prime}(u) \Lambda(s, u)\right]
$$

and

$$
\tilde{L}_{s}^{y} f(u)=f^{\prime}(u)^{T} m(s, y)+\frac{1}{2} \operatorname{tr}\left[\Lambda(s, y)^{T} f^{\prime \prime}(u) \Lambda(s, y)\right]
$$

We put

$$
H(s, t, u, v)=\left(L_{s}-\tilde{L}_{s}^{v}\right) f(u),
$$

where $f(u)=\tilde{p}(s, t, u, v)$. Then

$$
\begin{aligned}
H(s, t, x, y)= & \frac{1}{2} \sum_{i, j=1}^{p}\left(\sigma_{i j}(s, x)-\sigma_{i j}(s, y)\right) \frac{\partial^{2} \tilde{p}(s, t, x, y)}{\partial x_{i} \partial x_{j}} \\
& +\sum_{i=1}^{p}\left(m_{i}(s, x)-m_{i}(s, y)\right) \frac{\partial \tilde{p}(s, t, x, y)}{\partial x_{i}} .
\end{aligned}
$$

Now we define the convolution $\otimes$ :

$$
(f \otimes g)(s, t, u, v)=\int_{s}^{t} d \sigma \int_{\mathbb{R}^{p}} f(s, \sigma, u, w) g(\sigma, t, w, v) d w .
$$

We write $g \otimes H^{(0)}$ for $g$ and for $r=1,2, \ldots$ we denote the $r$-fold convolution $(g \otimes$ $\left.H^{(r-1)}\right) \otimes H$ by $g \otimes H^{(r)}$. With these notations we can formulate our next lemmas. Proofs of the first two lemmas can be found in McKean and Singer (1967). For a more detailed proof of Lemma 5.3 see also Il'in, Kalashnikov and Oleinik (1962).

Lemma 5.2 For $0 \leq s<t \leq 1$ the following formula holds:

$$
p(s, t, u, v)=\sum_{r=0}^{\infty}\left(\tilde{p} \otimes H^{(r)}\right)(s, t, u, v) .
$$

Lemma 5.3 There exist constants $C$ and $C_{1}$ (that do not depend on $x$ and $y$ ) such that the following inequalities hold:

$$
|H(s, t, x, y)| \leq C_{1} \rho^{-1} \phi_{C, \rho}(y-x)
$$

and

$$
\left|\tilde{p} \otimes H^{(r)}(s, t, x, y)\right| \leq C_{1}^{r+1} \frac{\rho^{r}}{\Gamma\left(1+\frac{r}{2}\right)} \phi_{C, \rho}(y-x),
$$

where $\rho^{2}=t-s, \phi_{C, \rho}(u)=\rho^{-p} \phi_{C}(u / \rho)$ and

$$
\phi_{C}(u)=\frac{\exp \left(-C\|u\|^{2}\right)}{\int \exp \left(-C\|v\|^{2}\right) d v}
$$


Lemma 5.4 There exist constants $C$ and $C_{1}$ such that the following estimate holds

$$
\left|\frac{\partial H(s, t, x, y)}{\partial s}\right| \leq C_{1} \rho^{-3} \phi_{C, \rho}(y-x),
$$

where $\rho$ and $\phi_{C, \rho}$ are defined as in Lemma 5.3.

Proof of Lemma 5.4. By Assumption (A5), $\sigma_{i j}(s, x)$ and $m_{i}(s, x)$ have partial derivatives with respect to $s$ that are Lipschitz continuous with respect to $x$. Using (5.3), one sees that for the statement of the lemma it suffices to show for some constants $C_{1}^{\prime}$ and $C_{2}^{\prime}$ that

$$
\begin{aligned}
&\left|\frac{\partial^{2} \tilde{p}(s, t, x, y)}{\partial x_{i} \partial x_{j}}\right| \leq C_{1}^{\prime} \rho^{-2} \phi_{C_{2}^{\prime}, \rho}(y-x), \\
&\left|\frac{\partial}{\partial s} \frac{\partial^{2} \tilde{p}(s, t, x, y)}{\partial x_{i} \partial x_{j}}\right| \leq C_{1}^{\prime} \rho^{-4} \phi_{C_{2}^{\prime}, \rho}(y-x) .
\end{aligned}
$$

These claims follow from Assumption (A5) by taking partial derivatives of $\tilde{p}$, see (5.1).

Lemma 5.5 There exist constants $C_{1}$ and $C$ such that the following estimates hold for $1 \leq k \leq p$

$$
\begin{aligned}
&\left|\frac{\partial}{\partial y_{k}} H(s, t, x, y)+\frac{\partial}{\partial x_{k}} H(s, t, x, y)\right| \leq C_{1} \rho^{-1} \phi_{C, \rho}(y-x), \\
&\left|\frac{\partial}{\partial s} H(s, t, x, y)+\frac{\partial}{\partial t} H(s, t, x, y)\right| \leq C_{1} \rho^{-1} \phi_{C, \rho}(y-x),
\end{aligned}
$$

where $\rho$ and $\phi_{C, \rho}$ are defined as in Lemma 5.3.

Proof of Lemma 5.5. The statements of the lemma can be seen from the definition of $H(s, t, x, y)$, well-known properties of Gaussian densities and (A5).

Lemma 5.6 There exist constants $C_{1}$ and $C$ such that the following estimate holds

$$
\left|\frac{\partial \tilde{p} \otimes H^{(r)}(s, t, x, y)}{\partial t}\right| \leq C_{1}^{r+1} \frac{\rho^{r-2}}{\Gamma\left(1+\frac{r}{2}\right)} \phi_{C, \rho}(y-x),
$$

where $\rho$ and $\phi_{C, \rho}$ are defined as in Lemma 5.3.

Proof of Lemma 5.6. We will prove (5.6) for $r=1$ and the following recursion formula for $r \geq 2$

$$
\begin{aligned}
& \frac{\partial}{\partial t} \tilde{p} \otimes H^{(r)}(s, t, x, y) \\
& \quad=\int_{s}^{t} d \tau \int \frac{\partial}{\partial \tau}\left[\hat{p} \otimes H^{(r-1)}(s, \tau, x, z)\right] \cdot H(\tau, t, z, y) d z+R_{r}(s, t, x, y)
\end{aligned}
$$


where for some constants $C_{1}^{\prime}$ and $C_{2}^{\prime}$

$$
\left|R_{r}(s, t, x, y)\right| \leq \frac{\left[C_{1}^{\prime}\right]^{r}}{\Gamma\left(1+\frac{r}{2}\right)} \rho^{r} \phi_{C_{2}^{\prime}, \rho}(y-x) .
$$

These claims imply the statement of the lemma: iterating (5.7) we get (5.6).

We prove now (5.7) for $r \geq 2$. From (5.5) we have for fixed $\tau \in(s, t)$ and $r \geq 2$

$$
\begin{aligned}
& \frac{\partial}{\partial t}\left(\int \tilde{p} \otimes H^{(r-1)}(s, \tau, x, z) \cdot H(\tau, t, z, y) d z\right) \\
& =\int \tilde{p} \otimes H^{(r-1)}(s, \tau, x, z) \cdot \frac{\partial}{\partial t} H(\tau, t, z, y) d z \\
& =-\int \tilde{p} \otimes H^{(r-1)}(s, \tau, x, z) \cdot \frac{\partial}{\partial \tau} H(\tau, t, z, y) d z+ \\
& \quad+R_{r}(s, \tau, t, x, y)
\end{aligned}
$$

where

$$
\left|R_{r}(s, \tau, t, x, y)\right| \leq \frac{C_{1}^{r}(\tau-s)^{\frac{r-1}{2}} \cdot(t-\tau)^{-1 / 2}}{\Gamma\left(\frac{1+r}{2}\right)} \phi_{C_{2}, \rho}(y-x) .
$$

Note now that

$$
\begin{aligned}
\frac{\partial}{\partial \tau} \int \tilde{p} \otimes H^{(r-1)}(s, \tau, x, z) \cdot H(\tau, t, z, y) d z \\
=\quad \int \frac{\partial}{\partial \tau}\left[\tilde{p} \otimes H^{(r-1)}(s, \tau, x, z)\right] \cdot H(\tau, t, z, y) d z+ \\
\quad \int \tilde{p} \otimes H^{(r-1)}(s, \tau, x, z) \cdot \frac{\partial}{\partial \tau} H(\tau, t, z, y) d z
\end{aligned}
$$

Comparing (5.9) and (5.10) we get

$$
\begin{aligned}
& \frac{\partial}{\partial t} \int \tilde{p} \otimes H^{(r-1)}(s, \tau, x, z) \cdot H(\tau, t, z, y) d z+ \\
& \quad \frac{\partial}{\partial \tau} \int \tilde{p} \otimes H^{(r-1)}(s, \tau, x, z) \cdot H(\tau, t, z, y) d z \\
& =\int \frac{\partial}{\partial \tau}\left[\tilde{p} \otimes H^{(r-1)}(s, \tau, x, z)\right] \cdot H(\tau, t, z, y) d z+R_{r}(s, \tau, t, x, y) .
\end{aligned}
$$

Integrating (5.9) in $\tau$ we have from (5.11)

$$
\begin{aligned}
\int_{s}^{t} d \tau & \frac{\partial}{\partial t}\left(\int \tilde{p} \otimes H^{(r-1)}(s, \tau, x, z) \cdot H(\tau, t, z, y) d z\right)= \\
& \int_{s}^{t} d \tau \int \frac{\partial}{\partial \tau}\left[\tilde{p} \otimes H^{(r-1)}(s, \tau, x, z)\right] \cdot H(\tau, t, z, y) d z- \\
& \left.\int \tilde{p} \otimes H^{(r-1)}(s, \tau, x, z) H(\tau, t, z, y) d z\right|_{\tau=s} ^{\tau=t}+R_{r}(s, t, x, y),
\end{aligned}
$$


where $R_{r}(s, t, x, y)$ satisfies (5.8). Now (5.7) for $r \geq 2$ immediately follows from (5.12) if we take into account that for $r \geq 2$

$$
\left.\int \tilde{p} \otimes H^{(r-1)}(s, \tau, x, z) H(\tau, t, z, y) d z\right|_{\tau=s}=0
$$

and

$$
\begin{aligned}
\frac{\partial}{\partial t} \tilde{p} \otimes H^{(r)}(s, t, x, y)= & \int_{s}^{t} d \tau \frac{\partial}{\partial t}\left(\int \tilde{p} \otimes H^{(r-1)}(s, \tau, x, z) \cdot H(\tau, t, z, y) d z\right) \\
& +\left.\int \tilde{p} \otimes H^{(r-1)}(s, \tau, x, z) \cdot H(\tau, t, z, y) d z\right|_{\tau=t}
\end{aligned}
$$

For the statement of the lemma it remains to show that (5.6) holds for $r=1$. Denote $\Psi_{1}(s, t, x, y)=\tilde{p}(s, t, x, y)$ and

$$
\Psi_{r}(s, t, x, y)=\tilde{p} \otimes H^{(r-1)}(s, t, x, y) .
$$

We have to prove that there exist some constants $C_{1}$ and $C_{2}$ with

$$
\left|\frac{\partial \Psi_{2}(s, t, x, y)}{\partial t}\right| \leq C_{1} \rho^{-1} \phi_{C_{2}, \rho}(y-x) .
$$

Remind that

$$
\Psi_{2}(s, t, x, y)=\int_{s}^{t} d \tau \int \tilde{p}(s, \tau, x, \omega) H(\tau, t, \omega, y) d \omega
$$

where $H$ and $\tilde{p}$ have been defined in (5.3) or (5.1), respectively. The proof of (5.14) is rather simple but very lengthy. For the proof we plug in Taylor expansions of $\sigma_{i j}(\tau, \omega)$ and $m_{i}(\tau, \omega)$ and use the fact that for densities convolution and differentiation can be interchanged. We denote the elements of the matrix $C_{y}(s, t)$ by $c_{i j}(s, t)$, the elements of the vector $\gamma_{y}(s, t)$ are denoted by $\gamma_{1}(s, t), \ldots, \gamma_{p}(s, t)$. Furthermore the elements of $C_{y}^{-1}(s, t)$ are denoted by $c^{i j}(s, t)$. Let $c_{(i)}(s, t)$ and $c^{(i)}(s, t)$ be the $i$ - th row of $C_{y}(s, t)$ and $C_{y}^{-1}(s, t)$, respectively, $\langle.,$.$\rangle means the usual scalar product in \mathbb{R}^{p}$.

For our claim (5.14) it suffices for a fixed pair $(i, j)$ or for a fixed $i$, respectively, to show

$$
\begin{aligned}
\left|\frac{\partial \Psi_{2}^{i j}(s, t, x, y)}{\partial t}\right| \leq C_{1} \rho^{-1} \phi_{C_{2}, \rho}(y-x) \\
\left|\frac{\partial \Psi_{2}^{i}(s, t, x, y)}{\partial t}\right| \leq C_{1} \rho^{-1} \phi_{C_{2}, \rho}(y-x) .
\end{aligned}
$$

Here

$$
\begin{aligned}
\Psi_{2}^{i j}(s, t, x, y)= & \int_{s}^{t} d \tau \int \tilde{p}(s, \tau, x, \omega)\left(\sigma_{i j}(\tau, \omega)-\sigma_{i j}(\tau, y)\right) \\
& \frac{\partial^{2} \tilde{p}(s, t, x, y)}{\partial \omega_{i} \partial \omega_{j}} d \omega \\
\Psi_{2}^{i}(s, t, x, y)= & \int_{s}^{t} d \tau \int \tilde{p}(s, \tau, x, \omega)\left(m_{i}(\tau, \omega)-m_{i}(\tau, y)\right) \\
& \frac{\partial^{2} \tilde{p}(s, t, x, y)}{\partial \omega_{i} \partial \omega_{j}} d \omega .
\end{aligned}
$$


This follows from the additivity of differentiation and of the convolution $\otimes$. We will give only the proof for (5.15). The proof of (5.16) can be done by similar (slightly simpler) arguments.

Using (A5) and Taylor expansions for $\sigma_{i j}(\tau, \omega)$ we get

$$
\sigma_{i j}(\tau, \omega)-\sigma_{i j}(\tau, y)=\left\langle-\frac{\partial \sigma_{i j}(\tau, y)}{\partial y}, y-\omega-\gamma_{y}(\tau, t)\right\rangle-\left\langle\frac{\partial \sigma_{i j}(\tau, y)}{\partial y}, \gamma_{y}(\tau, t)\right\rangle+R_{\sigma}
$$

where

$$
R_{\sigma}=2 \sum_{|\nu|=2} \frac{(\omega-y)^{\nu}}{\nu !} \int_{0}^{1}(1-\lambda) D^{\nu} \sigma_{i j}(\tau, y+\lambda(\omega-y)) d \lambda
$$

Hence

$$
\begin{aligned}
& \left(\sigma_{i j}(\tau, \omega)-\sigma_{i j}(\tau, y)\right) \frac{\partial^{2} \tilde{p}(\tau, t, \omega, y)}{\partial \omega_{i} \partial \omega_{j}}=\left[\left\langle-\frac{\partial \sigma_{i j}(\tau, y)}{\partial y}, y-\omega-\gamma_{y}(\tau, t)\right\rangle\right. \\
& \quad\left\langle c^{(i)}(\tau, t), y-\omega-\gamma_{y}(\tau, t)\right\rangle\left\langle c^{(j)}(\tau, t), y-\omega-\gamma_{y}(\tau, t)\right\rangle \\
& \left.\quad+\left\langle\frac{\partial \sigma_{i j}(\tau, y)}{\partial y}, y-\omega-\gamma_{y}(\tau, t)\right\rangle c^{i j}(\tau, t)\right] \tilde{p}(\tau, t, \omega, y) \\
& \quad-\left\langle\frac{\partial \sigma_{i j}(\tau, y)}{\partial y}, \gamma_{y}(\tau, t)\right\rangle \cdot \tilde{p}(\tau, t, \omega, y) \cdot \\
& \quad\left[\left\langle c^{(i)}(\tau, t), y-\omega-\gamma_{y}(\tau, t)\right\rangle\left\langle c^{(j)}(\tau, t), y-\omega-\gamma_{y}(\tau, t)\right\rangle-c^{i j}(\tau, t)\right] \\
& \quad+R_{\sigma} \frac{\partial^{2} \tilde{p}(\tau, t, \omega, y)}{\partial \omega_{i} \partial \omega_{j}}
\end{aligned}
$$

where it has been used that

$$
\begin{aligned}
\frac{\partial^{2} \tilde{p}(\tau, t, \omega, y)}{\partial \omega_{i} \partial \omega_{j}}= & \tilde{p}(\tau, t, \omega, y) \cdot\left[\left\langle c^{(i)}(\tau, t), y-\omega-\gamma_{y}(\tau, t)\right\rangle\right. \\
& \left.\left\langle c^{(j)}(\tau, t), y-\omega-\gamma_{y}(\tau, t)\right\rangle-c^{i j}(\tau, t)\right] .
\end{aligned}
$$

In what follows we need also the expression for the third derivative of $\tilde{p}$. Direct calculations give

$$
\begin{aligned}
& \frac{\partial^{3} \tilde{p}(\tau, t, \omega, y)}{\partial \omega_{i} \partial \omega_{j} \partial \omega_{k}}=\tilde{p}(\tau, t, \omega, y)\left[\left\langle c^{(i)}(\tau, t), y-\omega-\gamma_{y}(\tau, t)\right\rangle\left\langle c^{(j)}(\tau, t), y-\omega-\gamma_{y}(\tau, t)\right\rangle\right. \\
& \quad\left\langle c^{(k)}(\tau, t), y-\omega-\gamma_{y}(\tau, t)\right\rangle-c^{i j}(\tau, t)\left\langle c^{(k)}(\tau, t), y-\omega-\gamma_{y}(\tau, t)\right\rangle \\
& \left.\quad-c^{i k}(\tau, t)\left\langle c^{(j)}(\tau, t), y-\omega-\gamma_{y}(\tau, t)\right\rangle-c^{j k}(\tau, t)\left\langle c^{(i)}(\tau, t), y-\omega-\gamma_{y}(\tau, t)\right\rangle\right] .
\end{aligned}
$$

Using the last expression and the identity $C(\tau, t) C^{-1}(\tau, t)=I$ we get for $1 \leq l \leq p$

$$
\begin{aligned}
& \left\langle c_{(l)}(\tau, t), \frac{\partial}{\partial \omega}\left(\frac{\partial^{2} \tilde{p}(\tau, t, \omega, y)}{\partial \omega_{i} \partial \omega_{j}}\right)\right\rangle \\
= & {\left[\left(y_{l}-\omega_{l}-\gamma_{l}(\tau, t)\right)\left\langle c^{(i)}(\tau, t), y-\omega-\gamma_{y}(\tau, t)\right\rangle\left\langle c^{(j)}(\tau, t), y-\omega-\gamma_{y}(\tau, t)\right\rangle\right.} \\
& \left.-c^{i j}(\tau, t)\left(y_{l}-\omega_{l}-\gamma_{l}(\tau, t)\right)\right] \tilde{p}(\tau, t, \omega, y)-\delta_{l i} \frac{\partial \tilde{p}(\tau, t, \omega, y)}{\partial \omega_{j}}-\delta_{l j} \frac{\partial \tilde{p}(\tau, t, \omega, y)}{\partial \omega_{i}} .
\end{aligned}
$$


We define now

$$
\begin{aligned}
\tilde{p}_{i}(s, t, x, y) & =\left.\frac{\partial}{\partial y_{i}} p_{i}(s, t, x, y, z)\right|_{z=y}, \\
\tilde{p}_{i j}(s, t, x, y) & =\left.\frac{\partial^{2}}{\partial y_{i} \partial y_{j}} p_{i}(s, t, x, y, z)\right|_{z=y}, \\
\tilde{p}_{i j k}(s, t, x, y) & =\left.\frac{\partial^{3}}{\partial y_{i} \partial y_{j} \partial y_{k}} p_{i}(s, t, x, y, z)\right|_{z=y}, \\
\tilde{p}_{i j k l}(s, t, x, y) & =\left.\frac{\partial^{4}}{\partial y_{i} \partial y_{j} \partial y_{k} \partial y_{l}} p_{i}(s, t, x, y, z)\right|_{z=y}
\end{aligned}
$$

where

$$
\begin{aligned}
\tilde{p}(s, t, x, y, z)= & (2 \pi)^{-p / 2}\left(\operatorname{det} C_{z}(s, t)\right)^{-1 / 2} \\
& \exp \left[-\frac{1}{2}\left\{y-x-\gamma_{z}(s, t)\right\}^{\prime} C_{z}(s, t)^{-1}\left\{y-x-\gamma_{z}(s, t)\right\}\right] .
\end{aligned}
$$

$C_{y}(s, t)$ and $\gamma_{y}(s, t)$ have been defined in (5.1). Note that $\tilde{p}(s, t, x, y, y)=\tilde{p}(s, t, x, y)$. We denote the vector $\left[\tilde{p}_{i j}(s, t, x, y)\right]_{j=1, \ldots, p}$ by $\tilde{p}_{i .}(s, t, x, y)$. Similarly, $\tilde{p}_{i j} .(s, t, x, y)$ denotes the vector $\left[\tilde{p}_{i j k}(s, t, x, y)\right]_{k=1, \ldots, p}$ and $\tilde{p}_{i j . .}(s, t, x, y)$ denotes the matrix $\left[\tilde{p}_{i j k l}(s, t, x, y)\right]_{k, l=1, \ldots, p}$. With

$$
\frac{\partial}{\partial \omega}\left(\frac{\partial^{2} \tilde{p}(\tau, t, \omega, y)}{\partial \omega_{i} \partial \omega_{j}}\right)=-\tilde{p}_{i j} .(\tau, t, \omega, y)
$$

we get from (5.20) and (5.22)

$$
\begin{aligned}
& \left(\sigma_{i j}(\tau, \omega)-\sigma_{i j}(\tau, y)\right) \frac{\partial^{2} \tilde{p}(\tau, t, \omega, y)}{\partial \omega_{i} \partial \omega_{j}}= \\
& \quad\left\langle\frac{\partial \bar{\sigma}_{i j}(\tau, y)}{\partial y}, \tilde{p}_{i j .}(\tau, t, \omega, y)\right\rangle+\frac{\partial \sigma_{i j}(\tau, y)}{\partial y_{i}} \tilde{p}_{j}(\tau, t, \omega, y) \\
& \quad+\frac{\partial \sigma_{i j}(\tau, y)}{\partial y_{j}} \tilde{p}_{i}(\tau, t, \omega, y)-\left\langle\frac{\partial \sigma_{i j}(\tau, y)}{\partial y}, \gamma_{y}(\tau, t)\right\rangle \tilde{p}_{i j}(\tau, t, \omega, y) \\
& \quad+R_{\sigma} \tilde{p}_{i j}(\tau, t, \omega, y)
\end{aligned}
$$

where

$$
\frac{\partial \bar{\sigma}_{i j}(\tau, y)}{\partial y}=c_{(1)}(\tau, t) \frac{\partial \sigma_{i j}(\tau, y)}{\partial y_{1}}+c_{(2)}(\tau, t) \frac{\partial \sigma_{i j}(\tau, y)}{\partial y_{2}}+\ldots+c_{(p)}(\tau, t) \frac{\partial \sigma_{i j}(\tau, y)}{\partial y_{p}}
$$

Now taking into account (5.23) we get

$$
\Psi_{2}^{i j}(\tau, t, \omega, y)=I+I I+I I I+I V+V
$$

where

$$
I=\int_{s}^{t} d \tau \int \tilde{p}(s, \tau, x, \omega)\left\langle\frac{\partial \bar{\sigma}_{i j}(\tau, y)}{\partial y}, \tilde{p}_{i j} .(\tau, t, \omega, y)\right\rangle d \omega
$$




$$
\begin{aligned}
I I & =\int_{s}^{t} d \tau \int \tilde{p}(s, \tau, x, \omega) \frac{\partial \sigma_{i j}(\tau, y)}{\partial y_{i}} \tilde{p}_{j}(\tau, t, \omega, y) d \omega \\
I I I & =\int_{s}^{t} d \tau \int \tilde{p}(s, \tau, x, \omega) \frac{\partial \sigma_{i j}(\tau, y)}{\partial y_{j}} \cdot \tilde{p}_{i}(\tau, t, \omega, y) d \omega \\
I V & =-\int_{s}^{t} d \tau \int \tilde{p}(s, \tau, x, \omega)\left\langle\frac{\partial \sigma_{i j}(\tau, y)}{\partial y}, \gamma_{y}(\tau, t)\right\rangle \tilde{p}_{i j}(\tau, t, \omega, y) d \omega \\
V & =\int_{s}^{t} d \tau \int \tilde{p}(s, \tau, x, \omega) R_{\sigma} \tilde{p}_{i j}(\tau, t, \omega, y) d \omega .
\end{aligned}
$$

We have to show that for some constants $C_{1}$ and $C_{2}$ the derivatives of these five terms with respect to $t$ can be bounded by

$$
C_{1} \rho^{-1} \phi_{C_{2}, \rho}(y-x)
$$

For the treatment of the first term $I$ note that $I=\sum_{k=1}^{p} I_{k}$, where

$$
I_{k}=\int_{s}^{t} d \tau\left(\sum_{l=1}^{p} c_{l k}(\tau, t) \frac{\partial \sigma_{i j}(\tau, y)}{\partial y_{l}}\right) \int \tilde{p}(s, \tau, x, \omega) \tilde{p}_{i j k}(\tau, t, \omega, y) d \omega .
$$

By (5.21) and simple properties of Gaussian densities we have

$$
I_{k}=\int_{s}^{t} d \tau\left(\sum_{l=1}^{p} c_{l k}(\tau, t) \frac{\partial \sigma_{i j}(\tau, y)}{\partial y_{l}}\right) \tilde{p}_{i j k}(\tau, t, \omega, y) .
$$

We argue now that $\left|d I_{k} / d t\right|$ can be bounded by (5.25). This follows from $c_{l k}(t, t)=0$, from the boundedness of the functions $\sigma_{l k}(t, y), m_{i}(t, y)$ and $\frac{\partial \sigma_{i j}(\tau, y)}{\partial y_{l}}$, and from the following estimates

$$
\left|c_{l k}(\tau, t)\right| \leq C \cdot(t-s),\left|\frac{d c^{i k}(s, t)}{d t}\right| \leq C(t-s)^{-2} .
$$

For an estimate of the second summand II in (5.24) note that

$$
\begin{aligned}
I I & =\int_{s}^{t} d \tau \int \tilde{p}(s, \tau, x, \omega) \frac{\partial \sigma_{i j}(\tau, y)}{\partial y_{i}} \cdot \tilde{p}_{j}(\tau, t, \omega, y) d \omega \\
& =\left.\int_{s}^{t} d \tau \frac{\partial \sigma_{i j}(\tau, y)}{\partial y_{i}} \frac{\partial}{\partial y_{j}}\left(\int \tilde{p}(s, \tau, x, \omega) \tilde{p}(\tau, t, \omega, y, z) d \omega\right)\right|_{z=y} \\
& =\left(\int_{s}^{t} d \tau \frac{\partial \sigma_{i j}(\tau, y)}{\partial y_{i}}\right) \tilde{p}(s, t, x, y) \cdot\left\langle-c^{(j)}(s, t), y-x-\gamma_{y}(s, t)\right\rangle .
\end{aligned}
$$

It can be shown by straight forward calculations that $|d I I / d t|$ can be bounded by (5.25). The third term III can be treated as the second term.

For the fourth term $I V$ in (5.24) we have

$$
\begin{aligned}
I V= & -\int_{s}^{t} d \tau\left\langle\frac{\partial \sigma_{i j}(\tau, y)}{\partial y}, \gamma_{y}(\tau, t)\right\rangle \int \tilde{p}(s, \tau, x, \omega) \tilde{p}_{i j}(\tau, t, \omega, y) d \omega \\
= & -\int_{s}^{t} d \tau\left\langle\frac{\partial \sigma_{i j}(\tau, y)}{\partial y}, \gamma_{y}(\tau, t)\right\rangle \tilde{p}(s, t, x, y) \\
& {\left[\left\langle c^{(i)}(s, t), y-x-\gamma_{y}(s, t)\right\rangle\left\langle c^{(j)}(s, t), y-x-\gamma_{y}(s, t)\right\rangle-c^{i j}(s, t)\right] . }
\end{aligned}
$$


Denoting the expression [...] in square brackets by $B(s, t, x, y)$ we get for some constants $C_{1}, \ldots, C_{8}$

$$
\begin{aligned}
\left|\frac{d I V}{d t}\right|= & \mid\left\langle\int_{s}^{t} \frac{\partial \sigma_{i j}(\tau, y)}{\partial y} d \tau, m(t, y)\right\rangle \tilde{p}(s, t, x, y) B(s, t, x, y) \\
& +\int_{s}^{t} d \tau\left\langle\frac{\partial \sigma_{i j}(\tau, y)}{\partial y}, \gamma_{y}(\tau, t)\right\rangle \frac{d}{d t}(\tilde{p}(s, t, x, y) \times B(s, t, x, y)) \mid \\
\leq & C_{1}(t-s) \cdot(t-s)^{-p / 2} \exp \left(-C_{2} \frac{|y-x|^{2}}{t-s}\right)(t-s)^{-1} \\
& +C_{3}(t-s)^{2} \cdot(t-s)^{-p / 2-1} \exp \left(-C_{4} \frac{|y-x|^{2}}{t-s}\right)(t-s)^{-1} \\
& +C_{5}(t-s)^{2}(t-s)^{-p / 2} \exp \left(-C_{6} \frac{|y-x|^{2}}{t-s}\right)(t-s)^{-2} \\
\leq & C_{7} \rho^{-1} \phi_{C_{8}, \rho}(y-x) .
\end{aligned}
$$

It remains now to estimate the last summand $V$ in (5.24). Substituting (5.19) in the integrand we have

$$
\begin{aligned}
V= & 2 \int_{0}^{1}(1-\lambda) \int_{s}^{t} d \tau \int \tilde{p}(s, \tau, x, \omega) \\
& \sum_{|\nu|=2} \frac{(\omega-y)^{\nu}}{\nu !} \tilde{p}_{i j}(\tau, t, \omega, y) D^{\nu} \sigma_{i j}(\tau, y+\lambda(\omega-y)) d \omega d \lambda \\
= & V I+V I I,
\end{aligned}
$$

where (with $B(\tau, t, \omega, y)$ as defined above)

$$
\begin{aligned}
V I= & 2 \sum_{|\nu|=2} \int_{0}^{1}(1-\lambda) \int_{s}^{t} d \tau \int \tilde{p}(s, \tau, x, \omega) \frac{(\omega-y)^{\nu}}{\nu !} \tilde{p}(\tau, t, \omega, y) \\
& B(\tau, t, \omega, y)\left(D^{\nu} \sigma_{i j}(\tau, y+\lambda(\omega-y))-D^{\nu} \sigma_{i j}(\tau, y)\right) d \omega d \lambda, \\
V I I= & 2 \sum_{|\nu|=2} \int_{0}^{1}(1-\lambda) \int_{s}^{t} d \tau \int \tilde{p}(s, \tau, x, \omega) \frac{(\omega-y)^{\nu}}{\nu !} \tilde{p}(\tau, t, \omega, y) \\
& B(\tau, t, \omega, y) D^{\nu} \sigma_{i j}(\tau, y) d \omega d \lambda .
\end{aligned}
$$

We consider now $d V I / d t$. For fixed $\nu,|\nu|=2$, the sequence

$$
\delta(\tau, t, \omega, y)=\tilde{p}(\tau, t, \omega, y)(\omega-y)^{\nu} B(\tau, t, \omega, y)
$$

is a $\delta$-sequence (with an appropriate normalizing constant) as $\tau \rightarrow t$. Therefore for $\Delta t \rightarrow 0$, see also Assumption (A5),

$$
\sum_{|\nu|=2} \int_{0}^{1}(1-\lambda) \int_{t-\Delta t}^{t} d \tau \int \ldots d \omega d \lambda=o(\Delta t) .
$$

We obtain

$$
\frac{d V I}{d t}=2 \sum_{|\nu|=2} \int_{0}^{1}(1-\lambda) \int_{s}^{t} d \tau \int \tilde{p}(s, \tau, x, \omega) \frac{(\omega-y)^{\nu}}{\nu !}
$$




$$
\begin{aligned}
& \left(\frac{\partial \tilde{p}(\tau, t, \omega, y)}{\partial t} B(\tau, t, \omega, y)+\tilde{p}(\tau, t, \omega, y) \frac{\partial B(\tau, t, \omega, y)}{\partial t}\right) \\
& \left(D^{\nu} \sigma_{i j}(\tau, y+\lambda(\omega-y))-D^{\nu} \sigma_{i j}(\tau, y)\right) d \omega d \lambda .
\end{aligned}
$$

With (A5), this gives with constants $C_{1}, \ldots, C_{6}$

$$
\begin{aligned}
\left|\frac{d V I}{d t}\right| \leq & C_{1} \int_{s}^{t} d \tau \int \tilde{p}(s, \tau, x, \omega)|\omega-y|^{2+\delta} \\
& {\left[(t-\tau)^{-p / 2-1} \exp \left(-C_{2} \frac{|y-\omega|^{2}}{t-\tau}\right)(t-\tau)^{-1}+\right.} \\
& \left.(t-\tau)^{-p / 2} \exp \left(-C_{3} \frac{|y-\omega|^{2}}{t-\tau}\right)(t-\tau)^{-2}\right] d \omega \\
\leq & C_{4} \int_{s}^{t}|t-\tau|^{-1+\delta / 2} d \tau(t-s)^{\delta / 2-p / 2} \exp \left(-C_{5} \frac{|y-x|^{2}}{t-s}\right) \\
\leq & C_{6} \delta^{-1}(t-s)^{\delta / 2-p / 2} \exp \left(-C_{5} \frac{|y-x|^{2}}{t-s}\right) .
\end{aligned}
$$

Remind that $\left|\gamma_{i}(\tau, t)\right| \leq C(t-s), i=1, \ldots, p$, so to get the desired estimate for $d V I I / d t$ it is enough to obtain the following estimate for any fixed $\nu$ with $|\nu|=2$

$$
\begin{aligned}
& \frac{d}{d t} \int_{s}^{t} d \tau \int \tilde{p}(s, \tau, x, \omega) \frac{\left(\omega-y-\gamma_{y}(\tau, t)\right)^{\nu}}{\nu !} \tilde{p}(\tau, t, \omega, y) B(\tau, t, \omega, y) D^{\nu} \sigma_{i j}(\tau, y) d \omega \\
& \quad \leq C_{1}(t-s)^{-p / 2} \exp \left(-C_{2} \frac{|y-x|^{2}}{t-s}\right)
\end{aligned}
$$

Denote the nonvanishing coordinates of $\nu$ by $r$ and $q, 1 \leq r \leq q \leq p$. It is straightforward to verify the following representation

$$
\begin{aligned}
& \left(\omega-y-\gamma_{y}(\tau, t)\right)^{\nu} \tilde{p}(\tau, t, \omega, y) B(\tau, t, \omega, y) \\
= & \left\langle\tilde{p}_{i j . .}(\tau, t, \omega, y) c_{(r)}(\tau, t), c_{(q)}(\tau, t)\right\rangle \\
& +c_{r q}(\tau, t) \tilde{p}_{i j}(\tau, t, \omega, y)+\delta_{i r}\left\langle\tilde{p}_{j .}(\tau, t, \omega, y), c_{(q)}(\tau, t)\right\rangle \\
& +\delta_{j r}\left\langle\tilde{p}_{i .}(\tau, t, \omega, y), c_{(q)}(\tau, t)\right\rangle+\delta_{i q}\left\langle\tilde{p}_{j .}(\tau, t, \omega, y), c_{(r)}(\tau, t)\right\rangle \\
& +\delta_{j q}\left\langle\tilde{p}_{i \cdot}(\tau, t, \omega, y), c_{(r)}(\tau, t)\right\rangle+\delta_{i r} \delta_{j q} \tilde{p}(\tau, t, \omega, y)+\delta_{j r} \delta_{i q} \tilde{p}(\tau, t, \omega, y),
\end{aligned}
$$

where $\delta_{i j}$ is Kronecker's delta. Now we substitute (5.29) into (5.28). Claim (5.28) follows by interchanging the order of differentiation and integration and by using bounds on the elements of the matrix $C_{y}$ and its derivatives. This shows that the bound (5.25) applies for $d V I I / d t$.

Lemma 5.7 The following bound holds:

$$
\left|D_{u}^{\nu} \tilde{p}_{n}\left(s_{n}(j), s_{n}(k), u, y\right)\right| \leq C \rho^{-|\nu|} \xi_{\rho}(y-u)
$$


for all $j, k, u$ and $y$ and for all $\nu$ with $0 \leq|\nu| \leq 2$. Here, $\rho=\left[s_{n}(k)-s_{n}(j)\right]^{1 / 2}[$ for simplicity the indices $n, j$ and $k$ are suppressed in the notation], $\xi_{\rho}(\bullet)=\rho^{-p} \xi(\bullet / \rho)$ and

$$
\xi(x)=\frac{\left[1+\|x\|^{S-2}\right]^{-1}}{\int\left[1+\|u\|^{S-2}\right]^{-1} d u} .
$$

The constant $S$ has been defined in Assumption (AD).

Proof of Lemma 5.7. We note first that $\tilde{p}_{n}\left(s_{n}(j), s_{n}(k), u, \bullet\right)$ is the density of the vector

$$
u+\mu_{j, k}+\sum_{i=j}^{k-1} \eta_{i},
$$

where $\mu_{j, k}=\sum_{i=j}^{k-1} \Delta_{n}(i+1) m\left\{s_{n}(i), y\right\}$ is deterministic, $\eta_{i}=\left[\Delta_{n}(i+1)\right]^{1 / 2} \tilde{\varepsilon}_{n}(i+$ $1),[i=j, \ldots, k-1]$, and $\varepsilon_{n}(i+1)$ is a sequence of independent variables with densities $q\left(s_{n}(i), y, \bullet\right)$. Let $f_{n}(\bullet)$ be the density of the normalized sum $V_{j, k}^{-1 / 2} \sum_{i=j}^{k-1} \eta_{i}$ where

$$
V_{j, k}=\sum_{i=j}^{k-1} \Delta_{n}(i+1) \Sigma\left(s_{n}(i), y\right)
$$

It follows from (A3) that for some constants $c_{1}, \ldots, c_{4}>0$ the following inequalities hold for all $\theta$ with $\|\theta\|=1$ and all $j<k$

$$
c_{1} \rho^{-1} \leq\left\langle V_{j, k}^{-1 / 2} \theta, \theta\right\rangle \leq c_{2} \rho^{-1}
$$

and

$$
c_{3} \rho^{-p} \leq \operatorname{det} V_{j, k}^{-1 / 2} \leq c_{4} \rho^{-p}
$$

Clearly, we have

$$
\tilde{p}_{n}\left(s_{n}(j), s_{n}(k), u, \bullet\right)=\operatorname{det} V_{j, k}^{-1 / 2} f_{n}\left\{V_{j, k}^{-1 / 2}\left(\bullet-u-\mu_{j, k}\right)\right\} .
$$

It follows from (A2) that an Edgeworth expansion holds for $f_{n}$. This implies the following expansion for $\tilde{p}_{n}\left(s_{n}(j), s_{n}(k), u, \bullet\right)$ because of (5.31) and (5.32).

$$
\begin{aligned}
& \tilde{p}_{n}\left(s_{n}(j), s_{n}(k), u, \bullet\right) \\
& =\operatorname{det} V_{j, k}^{-1 / 2}\left[\sum_{r=0}^{S-3}(k-j)^{-r / 2} P_{r}\left(-\phi:\left\{\bar{\chi}_{\beta, r}\right\}\right)\left(V_{j, k}^{-1 / 2}\left[\bullet-u-\mu_{j, k}\right]\right)\right. \\
& \left.\quad+O\left([k-j]^{-(S-2) / 2}\left[1+\left\|V_{j, k}^{-1 / 2}\left(\bullet-u-\mu_{j, k}\right)\right\|^{S}\right]^{-1}\right)\right]
\end{aligned}
$$

with standard notation, see Bhattacharya and Rao (1976), p. 53. In particular, $P_{r}$ denotes a product of a standard normal density with a polynomial that has coefficients depending only on cumulants of order $\leq r+2$. Expansion (5.33) can be proved along 
the lines of the proof of Theorem 19.3 in Bhattacharya and Rao (1976). Hence, for $C$ large enough it holds that

$$
\tilde{p}_{n}\left(s_{n}(j), s_{n}(k), u, \bullet\right) \leq C \xi_{\rho}(\bullet-u) .
$$

For seeing this note that for all $c$ there exists a constant $C^{\prime}$ with

$$
\exp \left(-c\|x\|^{2}\right) \leq C^{\prime} \frac{1}{1+\|x\|^{S}}
$$

This shows the lemma for $|\nu|=0$. For $|\nu|=1,2$ one proceeds as in the proof of Theorem 19.3 in Bhattacharya and Rao (1976) to obtain Edgeworth expansions for $\mathrm{D}_{u}^{\nu} \hat{p}_{n}\left(s_{n}(j), s_{n}(k), u, y\right)$. Proceeding as above one gets $(5.30)$.

In the next lemma we compare the infinitesimal operators $A_{n, j}$ and $\tilde{A}_{n, j}^{v}$ with the differential operators $L_{s}$ and $\tilde{L}_{s}^{v}$. We give a bound for the error if, in the definition of $H_{n}$, the terms $A_{n, j}$ and $\tilde{A}_{n, j}^{v}$ are replaced by $L_{s}$ or $\tilde{L}_{s}^{v}$, respectively.

Lemma 5.8 The following bound holds with a constant $C$

$$
\begin{aligned}
& \left|H_{n}\left(s_{n}(j), s_{n}(k), u, v\right)-K_{n}\left(s_{n}(j), s_{n}(k), u, v\right)-M_{n}\left(s_{n}(j), s_{n}(k), u, v\right)\right| \\
& \quad \leq C \Delta_{\max }^{1 / 2} \rho^{-1} \zeta_{\rho}(v-u)
\end{aligned}
$$

for all $j<k, u$ and $v$. Here $\rho$ denotes the term $\rho=\left[s_{n}(k)-s_{n}(j)\right]^{1 / 2}$. We write $\zeta_{\rho}(\bullet)=\rho^{-p} \zeta(\bullet / \rho)$ where

$$
\zeta(x)=\frac{\left[1+\|x\|^{S-4}\right]^{-1}}{\int\left[1+\|u\|^{S-4}\right]^{-1} d u} .
$$

For $j<k-1$ the function $K_{n}$ is defined as

$$
K_{n}\left(s_{n}(j), s_{n}(k), u, v\right)=\left(L_{s_{n}(j)}-\tilde{L}_{s_{n}(j)}^{v}\right) f(u),
$$

where $f(u)=\tilde{p}_{n}\left(s_{n}(j), s_{n}(k), u, v\right)$. Furthermore, for $j<k-1$ we define

$$
\begin{gathered}
M_{n}\left(s_{n}(j), s_{n}(k), u, v\right)=3 \Delta_{n}(j+1)^{1 / 2} \sum_{|\nu|=3} \sum_{|\mu|=1} \int_{\mathbb{R}^{p}} \int_{0}^{1} D_{v}^{\mu} q\left(s_{n}(j), v, \theta\right)(v-u)^{\mu} \\
\frac{\theta^{\nu}}{\nu !} D_{u}^{\nu} \tilde{p}_{n}\left(s_{n}(j+1), s_{n}(k), u+\delta \theta \Delta_{n}(j+1)^{1 / 2}, v\right)(1-\delta)^{2} d \delta d \theta .
\end{gathered}
$$

For $j=k-1$ we define

$$
K_{n}\left(s_{n}(j), s_{n}(k), u, v\right)=M_{n}\left(s_{n}(j), s_{n}(k), u, v\right)=0
$$

Proof of Lemma 5.8. For $j=k-1$ note that $H_{n}\left(s_{n}(j), s_{n}(k), u, v\right)=0$. So it remains to consider the case $j<k-1$. Note first that [see (5.2)]

$$
H_{n}\left(s_{n}(j), s_{n}(k), u, v\right)=H_{n}^{1}\left(s_{n}(j), s_{n}(k), u, v\right)-H_{n}^{2}\left(s_{n}(j), s_{n}(k), u, v\right),
$$


where

$$
\begin{aligned}
& H_{n}^{1}\left(s_{n}(j), s_{n}(k), u, v\right)=\Delta_{n}(j+1)^{-1} \int p_{n, j}(u, w) \\
& \quad\left[\tilde{p}_{n}\left(s_{n}(j+1), s_{n}(k), w, v\right)-\tilde{p}_{n}\left(s_{n}(j+1), s_{n}(k), u, v\right)\right] d w
\end{aligned}
$$

and

$$
\begin{aligned}
& H_{n}^{2}\left(s_{n}(j), s_{n}(k), u, v\right)=\Delta_{n}(j+1)^{-1} \int \tilde{p}_{n, j}^{v}(u, w) \\
& \quad\left[\hat{p}_{n}\left(s_{n}(j+1), s_{n}(k), w, v\right)-\tilde{p}_{n}\left(s_{n}(j+1), s_{n}(k), u, v\right)\right] d w .
\end{aligned}
$$

On the right hand side of (5.36) we use now the substitution $\theta=\Delta_{n}(j+1)^{-1 / 2}(w-$ $u)-\Delta_{n}(j+1)^{1 / 2} m\left\{s_{n}(j), u\right\}$. With the notation $\lambda(w)=\tilde{p}_{n}\left(s_{n}(j+1), s_{n}(k), w, v\right)$ and $h(\theta)=m\left(s_{n}(j), u\right) \Delta_{n}(j+1)+\theta \Delta_{n}(j+1)^{1 / 2}$ this gives

$$
H_{n}^{1}\left(s_{n}(j), s_{n}(k), u, v\right)=\Delta_{n}(j+1)^{-1} \int q\left(s_{n}(j), u, \theta\right)[\lambda\{u+h(\theta)\}-\lambda(u)] d \theta .
$$

Remind that $q\left(s_{n}(j), u, \bullet\right)$ denotes the conditional density of $\varepsilon_{n}(j+1)$. We use now the expansion

$$
\lambda\{u+h(\theta)\}-\lambda(u)=\sum_{1 \leq|\nu| \leq 2} \frac{h(\theta)^{\nu}}{\nu !}\left(\mathrm{D}^{\nu} \lambda\right)(u)+3 \sum_{|\nu|=3} \frac{h(\theta)^{\nu}}{\nu !} \int_{0}^{1}(1-\delta)^{2}\left(\mathrm{D}^{\nu} \lambda\right)\{u+\delta h(\theta)\} d \delta .
$$

Using now that $\varepsilon_{n}(j)$ has conditional mean 0 we get that

$$
\begin{aligned}
& H_{n}^{1}\left(s_{n}(j), s_{n}(k), u, v\right) \\
& =\lambda^{\prime}(u)^{T} m\left(s_{n}(j), u\right)+\frac{1}{2} \operatorname{tr}\left[\Sigma\left\{s_{n}(j), u\right\} \lambda^{\prime \prime}(u)\right] \\
& \quad+\Delta_{n}(j+1) \sum_{|\nu|=2} \frac{m\left(s_{n}(j), u\right)^{\nu}}{\nu !}\left(\mathrm{D}^{\nu} \lambda\right)(u)+3 \sum_{|\nu|=3} \Delta_{n}(j+1)^{-1} \\
& \quad \iint_{0}^{1} q\left(s_{n}(j), u, \theta\right) \frac{h(\theta)^{\nu}}{\nu !}(1-\delta)^{2}\left(\mathrm{D}^{\nu} \lambda\right)\{u+\delta h(\theta)\} d \delta d \theta .
\end{aligned}
$$

Note that the first two terms on the right hand side of (5.38) are equal to $L_{s} f(u)$ with $f(u)=\tilde{p}_{n}(s, t, u, v), s=s_{n}(j+1)$ and $t=s_{n}(k)$.

We treat now the term $H_{n}^{2}\left(s_{n}(j), s_{n}(k), u, v\right)$. On the right hand side of (5.37) we use the substitution $\theta=\Delta_{n}(j+1)^{-1 / 2}(w-u)-\Delta_{n}(j+1)^{1 / 2} m\left\{s_{n}(j), v\right\}$. With the notation $\tilde{h}(\theta)=m\left(s_{n}(j), v\right) \Delta_{n}(j+1)+\theta \Delta_{n}(j+1)^{1 / 2}$ and $f(u)=\tilde{p}_{n}(s, t, u, v)$ this gives

$$
\begin{aligned}
& H_{n}^{2}\left(s_{n}(j), s_{n}(k), u, v\right) \\
& =\quad \tilde{L}_{s}^{v} f(u)+\Delta_{n}(j+1) \sum_{|\nu|=2} \frac{m\left(s_{n}(j), v\right)^{\nu}}{\nu !}\left(\mathrm{D}^{\nu} \lambda\right)(u) \\
& \quad+3 \sum_{|\nu|=3} \Delta_{n}(j+1)^{-1} \\
& \quad \iint_{0}^{1} q\left(s_{n}(j), v, \theta\right) \frac{\tilde{h}(\theta)^{\nu}}{\nu !}(1-\delta)^{2}\left(\mathrm{D}^{\nu} \lambda\right)\{u+\delta \tilde{h}(\theta)\} d \delta d \theta .
\end{aligned}
$$


It remains to show that there exists a constant $C$ with

$$
\Delta_{n}(j+1)\left|m\left(s_{n}(j), u\right)^{\nu}-m\left(s_{n}(j), v\right)^{\nu}\right|\left|\left(\mathrm{D}^{\nu} \lambda\right)(u)\right| \leq C \Delta_{\max } \rho^{-1} \zeta_{\rho}(v-u)
$$

for $\nu$ with $|\nu|=2$ and

$$
\begin{aligned}
& \mid \Delta_{n}(j+1)^{-1} \iint_{0}^{1}\left[q\left(s_{n}(j), v, \theta\right) \tilde{h}(\theta)^{\nu}\left(\mathrm{D}^{\nu} \lambda\right)\{u+\delta \tilde{h}(\theta)\}\right. \\
& \left.\quad-q\left(s_{n}(j), u, \theta\right) h(\theta)^{\nu}\left(\mathrm{D}^{\nu} \lambda\right)\{u+\delta h(\theta)\}\right](1-\delta)^{2} d \delta d \theta \\
& \quad-M_{n}\left(s_{n}(j), s_{n}(k), u, v\right) \mid \\
& \leq C \Delta_{\max }^{1 / 2} \rho^{-1} \zeta_{\rho}(v-u)
\end{aligned}
$$

for $\nu$ with $|\nu|=3$.

Proof of (5.40). Because of assumption (A3) we have that for a constant $C$ it holds that $\left|m\left(s_{n}(j), u\right)^{\nu}-m_{n}\left(s_{n}(j), v\right)^{\nu}\right| \leq C\|u-v\|$. Claim (5.40) follows from Lemma 5.7, monotonicity of $\zeta(x)$ and $(\mathrm{A} 4)$.

Proof of (5.41). Note that for $|\nu|=3$

$$
\begin{aligned}
\max \left\{\left|\tilde{h}(\theta)^{\nu}\right|,\left|h(\theta)^{\nu}\right|\right\} & \leq C \Delta_{n}^{\frac{3}{2}}(j+1)(1+\|\theta\|)^{3}, \\
\left|\tilde{h}(\theta)^{\nu}-h(\theta)^{\nu}\right| & \leq C \Delta_{n}^{2}(j+1)(1+\|\theta\|)^{2}\|u-v\| .
\end{aligned}
$$

So for $|\nu|=3$ the left hand side of (5.41) does not exceed the following sum

$$
\begin{aligned}
& C \Delta_{n}^{\frac{1}{2}}(j+1) \int\|u-v\|^{2} \psi(\theta)(1+\|\theta\|)^{3}\left|\left(D^{\nu} \lambda\right)\{u+\delta h(\theta)\}\right| d \theta \\
& +C \Delta_{n}(j+1) \int\|u-v\| \psi(\theta)(1+\|\theta\|)^{2}\left|\left(D^{\nu} \lambda\right)\{u+\delta \tilde{h}(\theta)\}\right| d \theta \\
& +C \Delta_{n}^{\frac{1}{2}}(j+1) \int \psi(\theta)(1+\|\theta\|)^{3}\left|\left(D^{\nu} \lambda\right)\{u+\delta h(\theta)\}-\left(D^{\nu} \lambda\right)\{u+\delta \tilde{h}(\theta)\}\right| d \theta .
\end{aligned}
$$

We use now the following simple estimate. For an $\varepsilon>0$ suppose that $\|y\| \leq \varepsilon$. Then

$$
\frac{1}{1+\|x+y\|^{s}} \leq \frac{1}{1+[\|x\|-\varepsilon]^{s}} \leq \frac{1}{1+\left[\frac{\|x\|}{2}\right]^{s}} \leq \frac{2^{s}}{1+\|x\|^{s}}
$$

for $\|x\| \geq 2 \varepsilon$ and

$$
\frac{1}{1+\|x+y\|^{s}} \leq 1 \leq \frac{(2 \varepsilon)^{s}+1}{1+\|x\|^{s}}
$$

for $\|x\|<2 \varepsilon$. Hence,

$$
\frac{1}{1+\|x+y\|^{s}} \leq \frac{C(s, \varepsilon)}{1+\|x\|^{s}}
$$

with $C(s, \varepsilon)=\max \left\{2^{s},(2 \varepsilon)^{s}+1\right\}$ for all $x$. 
From assumptions (A2), (A4), (5.31), (5.32) and (5.33) it follows that for $|\nu|=3$

$$
\begin{aligned}
& \left|\left(D^{\nu} \lambda\right)\{u+\delta h(\theta)\}\right| \\
& \quad \leq c \rho^{-p-3}\left[1+\left\|\frac{v-u-\delta m\left(s_{n}(j), u\right) \Delta_{n}(j+1)-\theta \delta \Delta_{n}(j+1)^{\frac{1}{2}}}{\rho}\right\|^{s}\right]^{-1} .
\end{aligned}
$$

Similarly we get that

$$
\begin{aligned}
& \left|\left(D^{\nu} \lambda\right)\{u+\delta \tilde{h}(\theta)\}\right| \\
& \quad \leq c \rho^{-p-3}\left[1+\left\|\frac{v-u-\delta m\left(s_{n}(j), v\right) \Delta_{n}(j+1)-\theta \delta \Delta_{n}(j+1)^{\frac{1}{2}}}{\rho}\right\|^{s}\right]^{-1} .
\end{aligned}
$$

Applying (5.43) with $y=\left[\delta m\left(s_{n}(j), z\right) \Delta_{n}(j+1)+\theta \delta \Delta_{n}(j+1)^{\frac{1}{2}}\right] / \rho, z=u$ or $v$, and $\varepsilon=C \Delta_{n}(j+1)^{\frac{1}{2}}+\|\theta\|$ we get [note that $\left.\|y\| \leq \varepsilon\right]$ for $|\nu|=3$ with a constant $C(s)$ depending on $s$

$$
\max \left\{\left|\left(D^{\nu} \lambda\right)\{u+\delta h(\theta)\}\right|,\left|\left(D^{\nu} \lambda\right)\{u+\delta \tilde{h}(\theta)\}\right|\right\} \leq c \rho^{-p-3} \frac{C(s)\left(1+\|\theta\|^{s}\right)}{1+\left\|\frac{v-u}{\rho}\right\|^{s}}
$$

Note now that for $\nu$ with $|\nu|=4$ and for $\kappa$ with $|\kappa| \leq 1$ we have [because of $\mid \delta h(\theta)+$ $\left.\kappa \delta(h(\theta)-\tilde{h}(\theta)) \mid \leq C \Delta_{n}(j+1)+\|\theta\| \Delta_{n}(j+1)^{\frac{1}{2}}\right]$

$$
\left|\left(D^{\nu} \lambda\right)\{u+\delta h(\theta)+\kappa \delta(h(\theta)-\tilde{h}(\theta))\}\right| \leq c \rho^{-p-4} \frac{C(s)\left(1+\|\theta\|^{s}\right)}{1+\left\|\frac{v-u}{\rho}\right\|^{s}} .
$$

Furthermore we get for the difference in the integrand of the third term in (5.42) that

$$
\begin{aligned}
& \left|\left(D^{\nu} \lambda\right)\{u+\delta h(\theta)\}-\left(D^{\nu} \lambda\right)\{u+\delta \tilde{h}(\theta)\}\right| \\
& \quad \leq c \rho^{-p-4} \Delta_{n}(j+1)\|u-v\| \frac{C(s)\left(1+\|\theta\|^{s}\right)}{1+\left\|\frac{v-u}{\rho}\right\|^{s}} .
\end{aligned}
$$

Substituting (5.44), (5.46) into (5.42) and taking $s=S-3$ (see (A2)) we get that the left hand side of (5.41) does not exceed

$$
C \Delta_{\max }^{\frac{1}{2}} \rho^{-1} \zeta_{\rho}(v-u)
$$

Lemma 5.9 The following bound holds with a constant $C$

$$
\begin{aligned}
\left|K_{n}\left(s_{n}(j), s_{n}(k), u, v\right)\right| & \leq C \rho^{-1} \zeta_{\rho}(v-u), \\
\left|H_{n}\left(s_{n}(j), s_{n}(k), u, v\right)\right| & \leq C \rho^{-1} \zeta_{\rho}(v-u), \\
\left|M_{n}\left(s_{n}(j), s_{n}(k), u, v\right)\right| & \leq C \rho^{-1} \zeta_{\rho}(v-u),
\end{aligned}
$$

for all $j<k, u$ and $v$. Here again, $\rho=\left[s_{n}(k)-s_{n}(j)\right]^{1 / 2}$. The function $\zeta_{\rho}$ has been defined in Lemma 5.8 . 
Proof of Lemma 5.9. Note first that (5.48) follows from (5.47) with Lemma 5.8 and (A4). Claim (5.49) follows from the fact that $\Delta_{\max } \leq c \rho$ for a constant $c$ and from simple estimates. It remains to show (5.47). We have that

$$
\begin{aligned}
(5.50)\left|K_{n}\left(s_{n}(j), s_{n}(k), u, v\right)\right| \leq & \left|f^{\prime}(u)^{T}\left[m\left(s_{n}(j), u\right)-m\left(s_{n}(j), v\right)\right]\right| \\
& +\frac{1}{2} \operatorname{tr}\left\{\left[\Lambda\left(s_{n}(j), u\right)\right.\right. \\
& \left.\left.-\Lambda\left(s_{n}(j), v\right)\right] f^{\prime \prime}(u)\left[\Lambda\left(s_{n}(j), u\right)+\Lambda\left(s_{n}(j), v\right)\right]\right],
\end{aligned}
$$

where $f(u)=\tilde{p}_{n}\left(s_{n}(j+1), s_{n}(k), u, v\right)$. It follows from (A2) and (A3) that for $C^{\prime}$ large enough

$$
\left\|m\left(s_{n}(j), u\right)-m\left(s_{n}(j), v\right)\right\| \leq C^{\prime} \rho\left[\frac{\|v-u\|}{\rho}+1\right]
$$

and

$$
\left\|\Lambda\left(s_{n}(j), u\right)-\Lambda\left(s_{n}(j), v\right)\right\| \leq C^{\prime} \rho\left[\frac{\|v-u\|}{\rho}+1\right] .
$$

Now the lemma follows from Lemma 5.7, (5.50) - (5.52) and (A4).

Lemma 5.10 There exists a constant $C_{1}$ (that does not depend on $x$ and $y$ ) such that the following inequality holds:

$$
\left|\tilde{p}_{n} \otimes_{n} H_{n}^{(r)}\left(s_{n}(j), s_{n}(k), x, y\right)\right| \leq \frac{C_{1}^{r+1} \rho^{r}}{\Gamma\left(1+\frac{r}{2}\right)} \chi_{\rho}(y-x)
$$

for $0<j<k \leq n$, where

$$
\chi(x)=\frac{\left[1+\|x\|^{2 S^{\prime}-2}\right]^{-1}}{\int\left[1+\|u\|^{2 S^{\prime}-2}\right]^{-1} d u}
$$

and $\rho=\left[s_{n}(k)-s_{n}(j)\right]^{1 / 2}$.

Proof of Lemma 5.10. With the help of Lemmas 5.9 and 5.7 [note that $\xi / \zeta$ is bounded] we get

$$
\begin{aligned}
& \left|\tilde{p}_{n} \otimes_{n} H_{n}\left(s_{n}(j), s_{n}(k), x, z\right)\right| \\
& \quad \leq \sum_{i=j=0}^{k-1} \Delta_{n}(j+1) \int_{\mathbb{R}^{p}} \tilde{p}_{n}\left(s_{n}(j), s_{n}(i), x, v\right)\left|H_{n}\left(s_{n}(i), s_{n}(k), v, z\right)\right| d v \\
& \quad \leq C^{2} \sum_{i=j}^{k-1} \Delta_{n}(i+1)\left[s_{n}(k)-s_{n}(i)\right]^{-1} \zeta^{2, j, k}(z-x),
\end{aligned}
$$

where we put

$$
\zeta^{l, j, k}(x)=\max \left\{\zeta_{\rho_{1}} * \ldots * \zeta_{\rho_{l}}(x): \rho_{1} \geq 0, \ldots, \rho_{l} \geq 0, \rho_{1}^{2}+\ldots+\rho_{l}^{2}=\rho^{2}\right\} .
$$


Here $\zeta_{0}$ denotes the $\delta$-function. We use now that $\sum_{i=j}^{k-1} \Delta_{n}(i+1)\left[s_{n}(k)-s_{n}(i)\right]^{-1 / 2} \leq$ $\int_{s_{n}(j)}^{s_{n}(k)}\left[s_{n}(k)-v\right]^{-1 / 2} d v=\rho B\left(1, \frac{1}{2}\right)$, where $B(\alpha, \beta)=\int_{0}^{1} t^{\alpha-1}(1-t)^{\beta-1} d t$ is the beta function. We get

$$
\left|\tilde{p}_{n} \otimes_{n} H_{n}\left(s_{n}(j), s_{n}(k), x, z\right)\right| \leq C^{2} \rho B\left(1, \frac{1}{2}\right) \zeta^{2, j, k}(z-x) .
$$

Using (5.54) and (5.48) we get

$$
\begin{aligned}
\mid \tilde{p}_{n} \otimes_{n} & H_{n}^{(2)}\left(s_{n}(j), s_{n}(k), x, z\right) \mid \\
\leq & \sum_{i=j}^{k-1} \Delta_{n}(i+1) \int_{\mathbb{R}^{p}}\left|\tilde{p}_{n} \otimes_{n} H_{n}\left(s_{n}(j), s_{n}(i), x, v\right)\right| \\
& \left|H_{n}\left(s_{n}(i), s_{n}(k), v, z\right)\right| d v \\
\leq & C^{3} \rho^{2} \zeta^{3, j, k}(z-x) B\left(1, \frac{1}{2}\right) B\left(\frac{3}{2}, \frac{1}{2}\right),
\end{aligned}
$$

where it has been used that $\int_{s_{n}(j)}^{s_{n}(k)}\left[s_{n}(k)-v\right]^{1 / 2}\left[v-s_{n}(j)\right]^{-1 / 2} d v=\rho^{2} B\left(\frac{3}{2}, \frac{1}{2}\right)$. Using iteratively similar bounds we get

$$
\begin{aligned}
\left|\tilde{p}_{n} \otimes_{n} H_{n}^{(r)}\left(s_{n}(j), s_{n}(k), x, z\right)\right| & C^{r+1} \rho^{r} \zeta^{r+1, j, k}(z-x) B\left(1, \frac{1}{2}\right) \\
& B\left(\frac{3}{2}, \frac{1}{2}\right) \times \ldots \times B\left(\frac{r+1}{2}, \frac{1}{2}\right) . \\
\leq & C^{r+1} \Gamma\left(\frac{1}{2}\right)^{r} \rho^{r} \zeta^{r+1, j, k}(z-x) \frac{1}{\Gamma\left(\frac{r}{2}+1\right)} .
\end{aligned}
$$

For the statement of the lemma it suffices to show that $\left[1+\|x / \rho\|^{2 S^{\prime}-2}\right] \rho^{p} \zeta^{r+1, j, k}(x)$ is bounded by $\left(C^{\prime}\right)^{r+1}$ for a constant $C^{\prime}$. For this purpose note that due to our choice of $S^{\prime}$, see Assumption (A2), with constants $C_{1}, C_{2}$

$$
\zeta(x)=\frac{C_{1}}{1+\|x\|^{2 p S^{\prime}}} \leq C_{2} \prod_{i=1}^{p} \lambda\left(x_{i}\right),
$$

where $\lambda(x)=\left[1+x^{2 S^{\prime}}\right]^{-1}\left\{\int\left[1+u^{2 S^{\prime}}\right]^{-1} d u\right\}^{-1}$. This shows that for $\rho_{1}, \ldots, \rho_{r+1}, \rho_{1}^{2}+\ldots+$ $\rho_{r+1}^{2}=\rho^{2}$,

$$
\zeta_{\rho_{1}} * \ldots * \zeta_{\rho_{r+1}}(x) \leq C_{2}^{r+1} \prod_{i=1}^{p} \eta\left(x_{i}\right),
$$

where $\eta(u)=\lambda_{\rho_{1}} * \ldots * \lambda_{\rho_{r+1}}(u)$. Let us denote the Fourier transform of a function $\gamma$ by $\hat{\gamma}(t)=\int \exp (i t u) \gamma(u) d u$. Furthermore, here $\|\bullet\|_{1}$ is the usual $L_{1}$-norm in $R^{1}$. We will show that

$$
\left\|\hat{\eta}^{*}\right\|_{1} \leq C_{3}^{r+1} \rho^{-1}
$$

where $\eta^{*}(u)=\left[1+(u / \rho)^{2 S^{\prime}-2}\right] \eta(u)$ and where $C_{3}$ is a constant that does not depend on the special choice of $\rho_{1}, \ldots, \rho_{r+1}$. [Note that the function $\eta^{*}$ is in $L_{1}\left(R^{1}\right)$, and that for this reason its Fourier transform is well defined.] 
From (5.57) we get by the Fourier Inversion Theorem

$$
|\eta(u)| \leq \frac{C^{r+1} \rho^{-1}}{1+(u / \rho)^{2 S^{\prime}-2}}
$$

Because of

$$
\prod_{i=1}^{p} \frac{1}{1+x_{i}^{2 S^{\prime}-2}} \leq \frac{C_{4}}{1+\|x\|^{2 S^{\prime}-2}}
$$

[with some constant $C_{4}$ ] we get therefore from (5.56) that

$$
\zeta^{r+1, j, k}(x) \leq C_{5}^{r+1} \rho^{-p} \frac{1}{1+\|x / \rho\|^{2 S^{\prime}-2}}
$$

with some constant $C_{5}$, i.e. (5.57) holds and the lemma is proved.

It remains to show claim $(5.57)$.

Proof of (5.57). Note first that

$$
\left\|\hat{\eta}^{*}\right\|_{1} \leq\|\hat{\eta}\|_{1}+\frac{1}{\rho^{2 S^{\prime}-2}}\left\|\hat{\eta}^{\left(2 S^{\prime}-2\right)}\right\|_{1}
$$

where $\hat{\eta}^{\left(2 S^{\prime}-2\right)}$ means the derivative of order $2 S^{\prime}-2$ of the Fourier transform $\hat{\eta}$ of $\eta$. We show now that

$$
\|\hat{\eta}\|_{1} \leq(r+1)^{1 / 2} \rho^{-1}\|\hat{\lambda}\|_{1} .
$$

For the proof of claim (5.59) note first that there exists an $i_{*}$ with $\rho_{i_{*}}^{2} \geq \rho^{2} /(r+1)$. We get the following inequality:

$$
\begin{aligned}
\int|\hat{\eta}(t)| d t & \leq \int\left|\hat{\lambda}\left(t \rho_{1}\right)\right| \cdot \ldots \cdot\left|\hat{\lambda}\left(t \rho_{l}\right)\right| d t \\
& \leq \int\left|\hat{\lambda}\left(t \rho_{i_{*}}\right)\right| d t \\
& \leq(r+1)^{1 / 2} \rho^{-1} \int|\hat{\lambda}(t)| d t
\end{aligned}
$$

Note now that $\int|\hat{\lambda}(t)| d t$ is bounded, see Lemma 5.9. This shows (5.59).

To estimate $\left\|\hat{\eta}^{\left(2 S^{\prime}-2\right)}\right\|_{1}$ note first that $\left|\hat{\lambda}_{\rho_{i}}(t)\right|=\left|\hat{\lambda}\left(\rho_{i} t\right)\right| \leq 1$ and

$$
\left|\hat{\lambda}_{\rho_{i}}^{(k)}(t)\right| \leq \rho_{i}^{k} \int|u|^{k} \lambda(u) d u<\infty
$$

for $k=1, \ldots, 2 S^{\prime}-2$. Furthermore, for $\sum_{i=1}^{r+1}=2 S^{\prime}-2$ we have with some constants $C_{6}$ and $C_{7}$

$$
\begin{aligned}
& \int\left|\hat{\lambda}_{\rho_{1}}^{\left(k_{1}\right)}(t)\right| \cdot \ldots \cdot\left|\hat{\lambda}_{\rho_{r+1}}^{\left(k_{r+1}\right)}(t)\right| d t \\
& \quad \leq C_{6}^{r} \prod_{i \neq i_{*}} \rho_{i}^{k_{i}} \int\left|\hat{\lambda}_{\rho_{i_{*}}}^{\left(k_{i_{*}}\right)}(t)\right| d t \\
& \quad \leq C_{6}^{r} \rho^{2 S^{\prime}-2-k_{i_{*}}} \int \rho_{i_{*}}^{k_{i_{*}}}\left|\hat{\lambda}^{\left(k_{i_{*}}\right)}(u)\right| \frac{d t}{\rho_{i_{*}}} \\
& \quad \leq C_{7}^{r+1} \rho^{2 S^{\prime}-3}\left\|\hat{\lambda}^{\left(k_{i_{*}}\right)}\right\|_{1} .
\end{aligned}
$$


Using Leibnitz formula for $\eta(u)=\lambda_{\rho_{1}} * \ldots * \lambda_{\rho_{r+1}}(u)$ we get the following estimate from (5.60) with a constant $C_{8}$

$$
\left\|\hat{\eta}^{\left(2 S^{\prime}-2\right)}\right\|_{1} \leq C_{8} \rho^{2 S^{\prime}-3}\left(\left\|\hat{\lambda}^{(1)}\right\|_{1}+\ldots+\left\|\hat{\lambda}^{\left(2 S^{\prime}-2\right)}\right\|_{1}\right) .
$$

It is well known that $\left\|\hat{\lambda}^{(q)}\right\|_{1}$ is uniformly bounded for $q=0,1, \ldots, 2 S^{\prime}-2$, see e.g. Lemma 1 in Gel'fand and Shilov (1958), p. 236. Claim (5.57) follows now from $(5.58)-(5.61)$.

Lemma 5.11 For $0 \leq j<k \leq n$ the following formula holds:

$$
p_{n}\left(s_{n}(j), s_{n}(k), u, v\right)=\sum_{r=0}^{k-j}\left(\tilde{p}_{n} \otimes_{n}\left(M_{n}+K_{n}\right)^{(r)}\right)\left(s_{n}(j), s_{n}(k), u, v\right)+R,
$$

where

$$
|R| \leq C \Delta_{\max }^{\frac{1}{2}} \chi_{\rho}(v-u)
$$

for some constant $C$. The function $\chi$ has been defined in Lemma 5.10. Here again $\rho=\left[s_{n}(k)-s_{n}(j)\right]^{1 / 2}$.

Proof of Lemma 5.11. By Lemma 5.1 we have that

$$
p_{n}\left(s_{n}(j), s_{n}(k), u, v\right)=\sum_{r=0}^{k-j}\left(\tilde{p}_{n} \otimes_{n} H_{n}^{(r)}\right)\left(s_{n}(j), s_{n}(k), u, v\right) .
$$

For $r=0$ we have that

$$
\left(\tilde{p}_{n} \otimes_{n} H_{n}^{(r)}\right)\left(s_{n}(j), s_{n}(k), u, v\right)=\left(\tilde{p}_{n} \otimes_{n}\left(M_{n}+K_{n}\right)^{(r)}\right)\left(s_{n}(j), s_{n}(k), u, v\right),
$$

by definition. For $r=1$ we have by Lemmas 5.7 and 5.8 that

$$
\left(\tilde{p}_{n} \otimes_{n} H_{n}^{(r)}\right)\left(s_{n}(j), s_{n}(k), u, v\right)=\left(\tilde{p}_{n} \otimes_{n}\left(M_{n}+K_{n}\right)^{(r)}\right)\left(s_{n}(j), s_{n}(k), u, v\right)+R_{1},
$$

where

$$
\begin{aligned}
\left|R_{1}\right| \leq & \sum_{i=j}^{k-1} \Delta_{n}(i+1) \int_{\mathbb{R}^{p}} \tilde{p}_{n}\left(s_{n}(j), s_{n}(i), u, w\right) \\
& \left|H_{n}-M_{n}-K_{n}\right|\left(s_{n}(i), s_{n}(k), w, v\right) d w \\
\leq & C^{2} \zeta^{2, j, k}(v-u) \Delta_{\text {max }}^{1 / 2} \sum_{i=j}^{k-1} \Delta_{n}(i+1) \rho^{-1}
\end{aligned}
$$

where the function $\zeta^{l, j, k}$ was defined in (5.53). For the proof of (5.62) we use Lemma 5.8. We apply now that

$$
\begin{aligned}
\sum_{i=j}^{k-1} \Delta_{n}(i+1)\left[s_{n}(k)-s_{n}(i)\right]^{-1 / 2} & \leq \int_{s_{n}(j)}^{s_{n}(k)}\left[s_{n}(k)-v\right]^{-1 / 2} d v \\
& =\rho B(1,1 / 2) .
\end{aligned}
$$


Therefore we get from (5.62) that

$$
\left|R_{1}\right| \leq C^{2} \zeta^{2, j, k}(v-u) \Delta_{\max }^{\frac{1}{2}} \rho B(1,1 / 2) .
$$

With similar arguments we get

$$
\left(\tilde{p}_{n} \otimes_{n} H_{n}^{(2)}\right)\left(s_{n}(j), s_{n}(k), u, v\right)=\left(\tilde{p}_{n} \otimes_{n}\left(M_{n}+K_{n}\right)^{(2)}\right)\left(s_{n}(j), s_{n}(k), u, v\right)+R_{2},
$$

where

$$
\left|R_{2}\right| \leq 2 C^{3} \zeta^{3, j, k}(v-u) \Delta_{\max }^{\frac{1}{2}} \rho^{2} B(1,1 / 2) B(3 / 2,1 / 2) .
$$

For arbitrary $r$ it holds that

$$
\left(\tilde{p}_{n} \otimes_{n} H_{n}^{(r)}\right)\left(s_{n}(j), s_{n}(k), u, v\right)=\left(\tilde{p}_{n} \otimes_{n}\left(M_{n}+K_{n}\right)^{(r)}\right)\left(s_{n}(j), s_{n}(k), u, v\right)+R_{r},
$$

where

$$
\left|R_{r}\right| \leq C_{1}^{r+1} \zeta^{r+1, j, k}(v-u) \Delta_{\max }^{\frac{1}{2}} \rho^{r} \frac{\Gamma(1 / 2)^{r}}{\Gamma([r+3] / 2)} .
$$

In the proof of Lemma 5.10 we have shown that

$$
\zeta^{r+1, j, k}(v-u) \leq C^{r+1} \rho^{-p} \frac{1}{1+\|(v-u) / \rho\|^{2 S^{\prime}-2}} .
$$

This gives

$$
p_{n}\left(s_{n}(j), s_{n}(k), u, v\right)=\sum_{r=0}^{k-j} \tilde{p}_{n} \otimes_{n}\left(M_{n}+K_{n}\right)^{(r)}\left(s_{n}(j), s_{n}(k), u, v\right)+R,
$$

where

$$
\begin{aligned}
{\left[1+\|(v-u) / \rho\|^{2 S^{\prime}-2}\right]|R| } & \leq \sum_{r=1}^{\infty}\left[1+\|(v-u) / \rho\|^{2 S^{\prime}-2}\right]\left|R_{r}\right| \\
& \leq \Delta_{\max }^{\frac{1}{2}} \rho^{-p} \sum_{r=1}^{\infty} \rho^{r} C_{2}^{r} \frac{\Gamma(1 / 2)^{r}}{\Gamma([r+3] / 2)}
\end{aligned}
$$

Because this is bounded by $C \Delta_{\max }^{\frac{1}{2}} \rho^{-p}$ for some constant $C$, this shows the statement of the lemma.

We come now to the proof of our theorem.

Proof of Theorem 2.1. From Lemmas 5.2 and 5.3 we get for sufficiently large $n$

$$
p(s, t, u, v)=\sum_{r=0}^{n}\left(\tilde{p} \otimes H^{(r)}\right)(s, t, u, v)+O\left(\Delta_{\max }^{1 / 2} \exp \left(-\frac{C\|v-u\|^{2}}{t-s}\right)\right) .
$$

Furthermore, Lemma 5.11 implies that

$$
\begin{aligned}
p_{n}(0,1, u, v)= & \sum_{r=0}^{n}\left(\tilde{p}_{n} \otimes_{n}\left(M_{n}+K_{n}\right)^{(r)}\right)(0,1, u, v) \\
& +O\left(\Delta_{\max }^{1 / 2} \frac{1}{1+\|v-u\|^{2 S^{\prime}-2}}\right) .
\end{aligned}
$$


Because of (5.63) and (5.64) for the statement of the theorem it remains to show that

$$
\begin{aligned}
& \left|\sum_{r=0}^{n}\left(\tilde{p} \otimes H^{(r)}(0,1, x, y)-\tilde{p}_{n} \otimes_{n}\left(M_{n}+K_{n}\right)^{(r)}(0,1, x, y)\right)\right| \\
& =O\left(\Delta_{\max }^{1 / 2} \frac{1}{1+\|v-u\|^{2 S^{\prime}-2}}\right) .
\end{aligned}
$$

For the proof of (5.65) note that

$$
\begin{aligned}
& \left|\sum_{r=0}^{n} \tilde{p} \otimes H^{(r)}(0,1, x, y)-\tilde{p}_{n} \otimes_{n}\left(M_{n}+K_{n}\right)^{(r)}(0,1, x, y)\right| \\
& \leq|| \sum_{r=0}^{n} \tilde{p} \otimes H^{(r)}(0,1, x, y)-\tilde{p} \otimes_{n} H^{(r)}(0,1, x, y) \mid \\
& \quad+\left|\sum_{r=0}^{n} \tilde{p} \otimes_{n} H^{(r)}(0,1, x, y)-\tilde{p} \otimes_{n}\left(M_{n}+H\right)^{(r)}(0,1, x, y)\right| \\
& \quad+\left|\sum_{r=0}^{n} \tilde{p} \otimes_{n}\left(M_{n}+H\right)^{(r)}(0,1, x, y)-\tilde{p} \otimes_{n}\left(M_{n}+K_{n}\right)^{(r)}(0,1, x, y)\right| \\
& \quad+\left|\sum_{r=0}^{n} \tilde{p} \otimes_{n}\left(M_{n}+K_{n}\right)^{(r)}(0,1, x, y)-\tilde{p}_{n} \otimes_{n}\left(M_{n}+K_{n}\right)^{(r)}(0,1, x, y)\right| \\
& =\quad T_{1}+T_{2}+T_{3}+T_{4} .
\end{aligned}
$$

For $T_{1}, T_{2}, T_{3}$ and $T_{4}$ we will show the following estimates

$$
T_{k}=O\left(\Delta_{\max }^{1 / 2} \frac{1}{1+\|y-x\|^{2 S^{\prime}-2}}\right),
$$

where $k=1, \ldots, 4$. This shows (5.65). It remains to show (5.67).

Proof of (5.67) for $k=1$. We have

$$
\begin{aligned}
T_{1} \leq & \sum_{r=1}^{n} \mid \int_{0}^{1} d s_{r} \int \Psi_{r}\left(0, s_{r}, x, v\right) H\left(s_{r}, 1, v, y\right) d v \\
& -\sum_{j=0}^{n-1} \Delta_{n}(j+1) \int \Psi_{r}\left(0, s_{n}(j), x, v\right) H\left(s_{n}(j), 1, v, y\right) d v \mid \\
& +\sum_{r=2}^{n}\left|\sum_{j=0}^{n-1} \Delta_{n}(j+1) \int\left(\Psi_{r}\left(0, s_{n}(j), x, v\right)-\Psi_{r}^{\Delta}\left(0, s_{n}(j), x, v\right)\right) H\left(s_{n}(j), 1, v, y\right) d v\right|
\end{aligned}
$$

where $\Psi_{r}$ is defined in (5.13) and where

$$
\begin{aligned}
& \Psi_{1}^{\Delta}\left(0, s_{n}(j), x, v\right)=\tilde{p}\left(0, s_{n}(i), x, v\right) \\
& \Psi_{r}^{\Delta}\left(0, s_{n}(j), x, v\right)=\sum_{i=0}^{j-1} \Delta_{n}(i+1) \int \Psi_{r-1}^{\Delta}\left(0, s_{n}(i), x, \omega\right) H\left(s_{n}(i), s_{n}(j), \omega, v\right) d \omega
\end{aligned}
$$

for $r \geq 2$. 
Denote $A_{r}(0,0, x, v)=0$ and

$$
\begin{aligned}
A_{r}\left(0, s_{n}(k), x, v\right)= & \int_{0}^{s_{n}(k)} d s_{r} \int \Psi_{r}\left(0, s_{r}, x, \omega\right) H\left(s_{r}, s_{n}(k), \omega, v\right) d \omega \\
& -\sum_{j=0}^{k-1} \Delta_{n}(j+1) \int \Psi_{r}\left(0, s_{n}(j), x, \omega\right) H\left(s_{n}(j), s_{n}(k), \omega, v\right) d \omega .
\end{aligned}
$$

Then we can rewrite our inequality in the form

$$
T_{1} \leq \sum_{r=1}^{n}\left|A_{r}(0,1, x, y)\right|+\sum_{r=2}^{n}\left|\left(\left(\Psi_{r}-\Psi_{r}^{\Delta}\right) \otimes_{n} H\right)(0,1, x, y)\right|
$$

Note that for $r \geq 2$

$$
\begin{aligned}
& \Psi_{r}\left(0, s_{n}(j), x, v\right)-\Psi_{r}^{\Delta}\left(0, s_{n}(j), x, v\right) \\
& \quad=A_{r-1}\left(0, s_{n}(j), x, v\right)+\left(\left(\Psi_{r-1}-\Psi_{r-1}^{\Delta}\right) \otimes_{n} H\right)\left(0, s_{n}(j), x, v\right)
\end{aligned}
$$

We apply now Lemma 5.6 to estimate $A_{r}\left(0, s_{n}(j), x, v\right)$. Let us consider the function

$$
\Lambda_{r}(\tau)=\int \Psi_{r}(0, \tau, x, \omega) H(\tau, s, \omega, v) d \omega .
$$

Let $\tau, \tau+\Delta \tau \in[0, s]$. We have by Lemmas $5.4,5.6$ and 5.3 for $\Delta \tau \geq 0$

$$
\begin{aligned}
\left|\Lambda_{r}(\tau+\Delta \tau)-\Lambda_{r}(\tau)\right| & \\
=\Delta & \tau\left|\iint_{0}^{1} \frac{\partial}{\partial \tau}\left[\Psi_{r}(0, \tau+h \Delta \tau, x, \omega) H(\tau+h \Delta \tau, s, \omega, v)\right] d h d \omega\right| \\
=\Delta \tau & \mid \int_{0}^{1} d h\left[\int H(\tau+h \Delta \tau, s, \omega, v) \frac{\partial}{\partial \tau} \Psi_{r}(0, \tau+h \Delta \tau, x, \omega)\right. \\
& \left.+\Psi_{r}(0, \tau+h \Delta \tau, x, \omega) \frac{\partial}{\partial \tau} H(\tau+h \Delta \tau, s, \omega, v) d \omega\right] \mid \\
\leq \Delta & \int_{0}^{1} d h\left\{\int C_{1}^{r} \frac{(\tau+h \Delta \tau)^{\frac{r-1}{2}-1-\frac{p}{2}}}{\Gamma\left(1+\frac{r-1}{2}\right)} \exp \left(-\frac{C_{2}|\omega-x|^{2}}{\tau+h \Delta \tau}\right)\right. \\
& \quad(s-\tau-h \Delta \tau)^{-\frac{p}{2}-\frac{1}{2}} \exp \left(-\frac{C_{3}|v-\omega|^{2}}{s-\tau-h \Delta \tau}\right) \\
& +C_{4}^{r} \frac{(\tau+h \Delta \tau)^{\frac{r-1}{2}-\frac{p}{2}}}{\Gamma\left(1+\frac{r-1}{2}\right)} \exp \left(-\frac{C_{5}|\omega-x|^{2}}{\tau+h \Delta \tau}\right) \\
& \left.C_{6}(s-\tau-h \Delta \tau)^{-\frac{p}{2}-\frac{3}{2}} \exp \left(-\frac{C_{7}|v-\omega|^{2}}{s-\tau-h \Delta \tau}\right) d \omega\right\} \\
\leq & C_{8}^{r} \Delta \tau \\
\Gamma\left(1+\frac{r-1}{2}\right) & s / 2 \exp \left(-\frac{C_{9}|v-x|^{2}}{s}\right) \int_{0}^{1} d h\left((s-\tau-h \Delta \tau)^{-\frac{3}{2}}+(\tau+h \Delta \tau)^{-\frac{3}{2}}\right) .
\end{aligned}
$$

This gives

$$
\begin{aligned}
& \left|\Lambda_{r}(\tau+\Delta \tau)-\Lambda_{r}(\tau)\right| \\
& \quad \leq \frac{C_{8}^{r}}{\Gamma\left(1+\frac{r-1}{2}\right)} s^{-p / 2} \exp \left(-\frac{C_{9}|v-x|^{2}}{s}\right)\left(\frac{\Delta \tau}{\tau^{3 / 2}}+\frac{\Delta \tau}{(s-\tau-\Delta \tau)^{3 / 2}}\right)
\end{aligned}
$$


and hence (with $s=s_{n}(k)$ )

$$
\begin{aligned}
& \left|\int_{s_{n}(j)}^{s_{n}(j+1)} \Lambda_{r}(\tau) d \tau-\Delta_{n}(j+1) \Lambda_{r}\left(s_{n}(j)\right)\right| \\
& \quad \leq \int_{s_{n}(j)}^{s_{n}(j+1)} \max _{\tau \in\left[s_{n}(j), s_{n}(j+1)\right]}\left|\Lambda_{r}(\tau)-\Lambda_{r}\left(s_{n}(j)\right)\right| d \tau \\
& \quad \leq \frac{C_{8}^{r}}{\Gamma\left(1+\frac{r-1}{2}\right)} s_{n}^{-p / 2}(k) \exp \left(-\frac{C_{9}|v-x|^{2}}{s_{n}(k)}\right)\left(\frac{\Delta_{n}^{2}(j+1)}{s_{n}^{3 / 2}(j)}+\frac{\Delta_{n}^{2}(j+1)}{\left(s_{n}(k)-s_{n}(j+1)\right)^{3 / 2}}\right) .
\end{aligned}
$$

Suppose now that $s_{n}(k) \geq 2 \Delta_{\text {max }}^{1 / 2}$. We put

$$
\begin{aligned}
B & =\left[0, \Delta_{\max }^{1 / 2}\right] \cup\left[s_{n}(k)-\Delta_{\max }, s_{n}(k)\right], \\
B_{n} & =\left\{j: 0 \leq s_{n}(j) \leq \Delta_{\max }^{1 / 2} \text { or } s_{n}(k)-\Delta_{\max } \leq s_{n}(j) \leq s_{n}(k)\right\} .
\end{aligned}
$$

Then

$$
\begin{aligned}
& \left|A_{r}\left(0, s_{n}(k), x, v\right)\right|=\left|\int_{0}^{s_{n}(k)} \Lambda_{r}(\tau) d \tau-\sum_{j=0}^{k-1} \Delta_{n}(j+1) \Lambda_{r}\left(s_{n}(j)\right)\right| \\
& \leq \int_{B}\left|\Lambda_{r}(\tau)\right| d \tau+\sum_{j \in B_{n}} \Delta_{n}(j+1)\left|\Lambda_{r}\left(s_{n}(j)\right)\right| \\
& \quad+\frac{C_{8}^{r}}{\Gamma\left(1+\frac{r-1}{2}\right)} s_{n}^{-p / 2}(k) \exp \left(-\frac{C_{9}|v-x|^{2}}{s_{n}(k)}\right)\left(S_{1}+S_{2}+S_{3}+S_{4}\right),
\end{aligned}
$$

where

$$
\begin{aligned}
& S_{1}=\sum_{\left\{j: \Delta_{\max }^{1 / 3} \leq s_{n}(j) \leq s_{n}(k)\right\}} \frac{\Delta_{n}^{2}(j+1)}{s_{n}^{3 / 2}(j)}, \\
& S_{2}=\sum_{\left\{j: \Delta_{\max }^{1 / 2} \leq s_{n}(j) \leq \Delta_{\max }^{1 / 3}\right\}} \frac{\Delta_{n}^{2}(j+1)}{s_{n}^{3 / 2}(j)}, \\
& S_{3}=\sum_{\left\{j: 0 \leq s_{n}(j+1) \leq s_{n}(k)-\Delta_{\max }^{1 / 3}\right\}} \frac{\Delta_{n}^{2}(j+1)}{\left(s_{n}(k)-s_{n}(j+1)\right)^{3 / 2}}, \\
& S_{4}=\sum_{\left\{j: s_{n}(k)-\Delta_{\max }^{1 / 3} \leq s_{n}(j+1) \leq s_{n}(k)-\Delta_{\max }\right\}} \frac{\Delta_{n}^{2}(j+1)}{\left(s_{n}(k)-s_{n}(j+1)\right)^{3 / 2}} .
\end{aligned}
$$

We have

$$
\begin{aligned}
S_{1} & \leq \Delta_{\max }^{-1 / 2} \Delta_{\max } s_{n}(k)=\Delta_{\max }^{1 / 2} s_{n}(k), \\
S_{2} & \leq \Delta_{\max }^{-3 / 4} \Delta_{\max } \Delta_{\max }^{1 / 3}=o\left(\Delta_{\max }^{1 / 2}\right), \\
S_{3} & \leq \Delta_{\max }^{-1 / 2} \Delta_{\max } s_{n}(k)=\Delta_{\max }^{1 / 2} s_{n}(k), \\
S_{4} & =\Delta_{\max } \sum_{\left\{j: s_{n}(k)-\Delta_{\max }^{1 / 3} \leq s_{n}(j+1) \leq s_{n}(k)-\Delta_{\max }\right\}} \frac{\Delta_{n}(j+1)}{\left(s_{n}(k)-s_{n}(j+1)\right)^{3 / 2}} \\
& \leq C \Delta_{\max } \int_{s_{n}(k)-\Delta_{\max }^{1 / 3}}^{s_{n}(k)-\Delta_{\max }}\left(s_{n}(k)-v\right)^{-3 / 2} d v \leq C_{1} \Delta_{\max }^{1 / 2} .
\end{aligned}
$$


From the estimates of Lemma 5.3 we obtain (remind that now $s_{n}(k) \geq 2 \Delta_{\max }^{1 / 2}$ )

$$
\begin{aligned}
\int_{B}\left|\Lambda_{r}(\tau)\right| d \tau \leq \frac{C_{1}^{r}}{\Gamma\left(1+\frac{r-1}{2}\right)} s_{n}^{-p / 2}(k) \exp \left(-\frac{C_{2}|v-x|^{2}}{s_{n}(k)}\right) & \left(\int_{0}^{\Delta_{\max }^{1 / 2}}\left(s_{n}(k)-\tau\right)^{-1 / 2} \tau^{(r-1) / 2} d \tau+\int_{s_{n}(k)-\Delta_{\max }}^{s_{n}(k)}\left(s_{n}(k)-\tau\right)^{-1 / 2} d \tau\right) \\
\leq & \frac{C_{1}^{r}}{\Gamma\left(1+\frac{r-1}{2}\right)} s_{n}^{-p / 2}(k) \exp \left(-\frac{C_{2}|v-x|^{2}}{s_{n}(k)}\right) \Delta_{\max }^{1 / 2} s_{n}(k)^{0 \wedge(r-3 / 2)} \\
\sum_{j \in B_{n}} \Delta_{n}(j+1)\left|\Lambda_{r}\left(s_{n}(j)\right)\right| & \frac{C_{1}^{r}}{\Gamma\left(1+\frac{r-1}{2}\right)} s_{n}^{-p / 2}(k) \exp \left(-\frac{C_{2}|v-x|^{2}}{s_{n}(k)}\right) \\
\leq & \times \sum_{j \in B_{n}} \Delta_{n}(j+1) \frac{s_{n}^{(r-1) / 2}(j)}{\left(s_{n}(k)-s_{n}(j)\right)^{1 / 2}} \\
\leq & \frac{C_{1}^{r}}{\Gamma\left(1+\frac{r-1}{2}\right)} s_{n}^{-p / 2}(k) \exp \left(-\frac{C_{2}|v-x|^{2}}{s_{n}(k)}\right) \sum_{j \in B_{n}} \Delta_{n}(j+1) \\
\leq & \frac{C_{3}^{r}}{\Gamma\left(1+\frac{r-1}{2}\right)} s_{n}^{-p / 2}(k) \exp \left(-\frac{C_{2}|v-x|^{2}}{s_{n}(k)}\right) \Delta_{\text {max }}^{1 / 2} s_{n}(k)^{0 \wedge(r-3 / 2)}
\end{aligned}
$$

We get now from $(5.70)-(5.76)$ for $r \geq 1$

$$
\begin{aligned}
& \left|A_{r}\left(0, s_{n}(k), x, v\right)\right| \\
& \leq \frac{C_{3}^{r}}{\Gamma\left(1+\frac{r-1}{2}\right)} s_{n}^{-p / 2}(k) \exp \left(-\frac{C_{2}|v-x|^{2}}{s_{n}(k)}\right) \Delta_{\max }^{1 / 2} s_{n}(k)^{0 \wedge(r-3 / 2)} .
\end{aligned}
$$

It follows from the inequalities of Lemma 5.3 that the same estimate (5.77) holds for $s_{n}(k) \leq 2 \Delta_{\max }^{1 / 2}$. Now, iterative application of (5.68) and (5.69) gives

$$
\begin{aligned}
& \sum_{r=2}^{n}\left|\left(\left(\Psi_{r}-\Psi_{r}^{\Delta}\right) \otimes_{n} H\right)(0,1, x, y)\right| \\
& \leq \sum_{r=1}^{\infty} \sum_{l=1}^{\infty}\left|\left(A_{r} \otimes_{n} H^{(l)}\right)(0,1, x, y)\right| .
\end{aligned}
$$

From (5.77) just as in Lemma 5.10 we obtain

$$
\begin{aligned}
& \sum_{r=1}^{\infty} \sum_{l=1}^{\infty}\left|\left(A_{r} \otimes_{n} H^{(l)}\right)(0,1, x, y)\right| \\
& \quad \leq\left(\sum_{r=1}^{\infty} \frac{C_{3}^{r}}{\Gamma\left(1+\frac{r}{2}\right)}\right)\left(\sum_{l=1}^{\infty} \frac{C_{4}^{l}}{\Gamma\left(1+\frac{l}{2}\right)}\right) \exp \left(-C_{5}(y-x)^{2}\right) \Delta_{\max }^{1 / 2}
\end{aligned}
$$

The desired estimate for $T_{1}$ follows from (5.68), (5.77), (5.78) and (5.79). 
Proof of (5.67) for $k=2$. For $r=1$ we have

$$
\begin{aligned}
& \tilde{p} \otimes_{n} H^{(r)}\left(0, s_{n}(k), x, y\right)-\tilde{p} \otimes_{n}\left(M_{n}+H\right)^{(r)}\left(0, s_{n}(k), x, y\right) \\
& =\tilde{p} \otimes_{n} M_{n}^{(r)}\left(0, s_{n}(k), x, y\right) \\
& =\sum_{j=0}^{k-1} \Delta_{n}(j+1)^{3 / 2} \sum_{|\mu|=1} \sum_{|\nu|=3} a_{\mu, \nu}(j),
\end{aligned}
$$

where

$$
\begin{aligned}
& a_{\mu, \nu}(j)=3 \int_{\mathbb{R}^{p}} \int_{\mathbb{R}^{p}} \int_{0}^{1} \tilde{p}\left(0, s_{n}(j), x, w\right) D_{y}^{\mu} q\left(s_{n}(j), y, \theta\right)(y-w)^{\mu} \\
& \frac{\theta^{\nu}}{\nu !} D_{w}^{\nu} \tilde{p}_{n}\left(s_{n}(j+1), s_{n}(k), w+\delta \theta \Delta_{n}(j+1)^{1 / 2}, y\right)(1-\delta)^{2} d \delta d \theta d w .
\end{aligned}
$$

We consider the index sets $J_{1}=\left\{j \leq k: s_{n}(j) \leq s_{n}(k) / 2\right\}$ and $J_{2}=\left\{j \leq k: s_{n}(j)>\right.$ $\left.s_{n}(k) / 2\right\}$ For $j \in J_{1}$ we get the following bound for $a_{\mu, \nu}(j)$ with constants $C_{1}, C_{2}$ and with $\kappa^{2}=s_{n}(k), \lambda^{2}=s_{n}(k)-s_{n}(j)$

$$
\begin{aligned}
\left|a_{\mu, \nu}(j)\right| & \leq C_{1} \int \tilde{p}\left(0, s_{n}(j), x, w\right) \lambda^{-2} \zeta_{\lambda}(y-w) d w \\
& \leq C_{2} \lambda^{-2} \zeta_{\kappa}(y-x) .
\end{aligned}
$$

This gives with a constant $C_{3}$

$$
\begin{aligned}
& \left|\sum_{j \in J_{1}} \Delta_{n}(j+1)^{3 / 2} \sum_{|\mu|=1} \sum_{|\nu|=3} a_{\mu, \nu}(j)\right| \\
& \leq C_{3} \sum_{j \in J_{1}} \Delta_{n}(j+1)^{3 / 2}\left[s_{n}(k)-s_{n}(j)\right]^{-1} \zeta_{\kappa}(y-x) \\
& \leq C_{3} \Delta_{\max }^{1 / 2} \zeta_{\kappa}(y-x) \int_{0}^{s_{n}(k) / 2}\left[s_{n}(k)-u\right]^{-1} d u \\
& \leq C_{3} \Delta_{\max }^{1 / 2} \zeta_{\kappa}(y-x)\left[\ln \left(s_{n}(k)\right)-\ln \left(s_{n}(k) / 2\right)\right] \\
& \leq C_{3} \ln (2) \Delta_{\max }^{1 / 2} \zeta^{2,0, k}(y-x) .
\end{aligned}
$$

We consider now $a_{\mu, \nu}(j)$ for $j \in J_{2}$. Denote the index $l$ with $\mu_{l}=1$ by $l(\mu)$. We consider first the case that $\nu_{l(\mu)}<3$. Then there exists an $l^{*} \neq l(\mu)$ with $\nu_{l^{*}} \geq 1$. Define $\nu_{l}^{*}=\nu_{l}$ for $l \neq l^{*}$ and $\nu_{l}^{*}=\nu_{l}-1$ for $l=l^{*}$. By integration by parts we get

$$
\begin{gathered}
a_{\mu, \nu}(j)=3 \int_{\mathbb{R}^{p}} \int_{\mathbb{R}^{p}} \int_{0}^{1} \frac{\partial}{\partial w_{l^{*}}} \tilde{p}\left(0, s_{n}(j), x, w\right) D_{y}^{\mu} q\left(s_{n}(j), y, \theta\right)(y-w)^{\mu} \\
\frac{\theta^{\nu}}{\nu !} D_{w}^{\nu^{*}} \tilde{p}_{n}\left(s_{n}(j+1), s_{n}(k), w+\delta \theta \Delta_{n}(j+1)^{1 / 2}, y\right)(1-\delta)^{2} d \delta d \theta d w .
\end{gathered}
$$

Using this equation we get the following bound for $a_{\mu, \nu}(j)$ with $\nu_{l(\mu)}<3$ ]

$$
\left|a_{\mu, \nu}(j)\right| \leq C_{4} \int \frac{\partial}{\partial w_{l^{*}}} \tilde{p}\left(0, s_{n}(j), x, w\right) \lambda^{-1} \zeta_{\lambda}(y-w) d w
$$

where $C_{4}$ is a constant and where again $\lambda^{2}=s_{n}(k)-s_{n}(j)$. 
By calculating $\partial / \partial w_{l^{*}} \tilde{p}\left(0, s_{n}(j), x, w\right)$ using the explicite definition (5.1) one can show that

$$
\left|a_{\mu, \nu}(j)\right| \leq C_{5} \iota^{-1} \lambda^{-1} \zeta^{2,0, k}(y-x),
$$

where $C_{5}$ is a constant and where $\iota^{2}=s_{n}(j)$ and again $\lambda^{2}=s_{n}(k)-s_{n}(j)$. For a definition of $\zeta^{2,0, k}$ see (5.53). For $a_{\mu, \nu}(j)$ with $\nu_{l(\mu)}=3$ note that after partial integration $a_{\mu, \nu}(j)$ is of the form

$$
\int f(z) z g^{(3)}(z) d z
$$

By integration by parts one gets under conditions on the tails of $f$ and $g$ that

$\int f(z) z g^{(3)}(z) d z=\int f(z)\left[(z g(z))^{(3)}-3 g^{(2)}(z)\right] d z=-\int f^{\prime}(z)\left[(z g(z))^{(2)}-3 g^{\prime}(z)\right] d z$.

By application of this equality one can show that for a constant $C_{6}$

$$
\left|a_{\mu, \nu}(j)\right| \leq C_{6}\left[\iota^{-2}+\iota^{-1} \lambda^{-1}\right] \zeta^{2,0, k}(y-x) .
$$

Application of these bounds gives for $j \in J_{2}$ with some constant $C_{7}$

$$
\begin{aligned}
& \left|\sum_{j \in J_{2}} \Delta_{n}(j+1)^{3 / 2} \sum_{|\mu|=1} \sum_{|\nu|=3} a_{\mu, \nu}(j)\right| \\
& \leq C_{7} \Delta_{\text {max }}^{1 / 2} s_{n}(k)^{-1 / 2} \zeta^{2,0, k}(y-x) .
\end{aligned}
$$

This gives that for $r=1$ it holds with some constant $C_{8}$

$$
\begin{aligned}
& \left|\tilde{p} \otimes_{n} H^{(r)}\left(0, s_{n}(k), x, y\right)-\tilde{p} \otimes_{n}\left(M_{n}+H\right)^{(r)}\left(0, s_{n}(k), x, y\right)\right| \\
& \leq C_{8} \Delta_{\text {max }}^{1 / 2} s_{n}(k)^{-1 / 2} \zeta^{2,0, k}(y-x) .
\end{aligned}
$$

We claim now that for $r \geq 1$ it holds that

$$
\begin{aligned}
& \left|\tilde{p} \otimes_{n} H^{(r)}\left(0, s_{n}(k), x, y\right)-\tilde{p} \otimes_{n}\left(M_{n}+H\right)^{(r)}\left(0, s_{n}(k), x, y\right)\right| \\
& \leq \frac{C_{8}^{r}}{\Gamma([r+2] / 2)} \Delta_{\text {max }}^{1 / 2} s_{n}(k)^{(r-2) / 2} \zeta^{r+1,0, k}(y-x) .
\end{aligned}
$$

This claim can be proved similarly as for the case $r=1$. An essential tool is Lemma 5.5. The first statement of this lemma implies the following bound

$$
\frac{\partial}{\partial w_{l}}\left(\tilde{p} \otimes_{n} H^{(s)}\right)\left(0, s_{n}(k), x, w\right) \leq \frac{C_{9}^{s+1} \kappa^{s-1} \zeta^{s+1,0, k}(w-x)}{\Gamma([s+1] / 2)}
$$

for $s<r$. This inequality can be shown by iterative application of integration by parts. With the help of this inequality and with Lemma 5.9 claim (5.80) follows with similar arguments as in the proof of Lemma 5.11.

Proof of (5.67) for $k=3$. First note that our conditions imply that (formal) differentiation with respect to $u$ up to second order is possible in both sides of (5.33). After calculations similar to the ones presented in the proof of Lemma 5.9 this gives

$$
\begin{gathered}
\left|H\left(s_{n}(i), s_{n}(k), u, y\right)-K_{n}\left(s_{n}(i), s_{n}(k), u, y\right)\right| \\
\leq C \Delta_{\max }^{1 / 2}\left(s_{n}(k)-s_{n}(i)\right)^{-1 / 2} \zeta_{\rho}(y-u) .
\end{gathered}
$$


Proceeding as in the proof of Lemma 5.10 we get with a constant $C$ [in the following arguments we will suppose that $C$ is sufficiently large]

$$
\begin{aligned}
\mid \tilde{p} & \otimes_{n}\left[\left(H+M_{n}\right)-\left(K_{n}+M_{n}\right)\right]\left(0, s_{n}(k), x, y\right) \mid \\
& \leq \sum_{j=0}^{k-1} \Delta_{n}(j+1) \int \tilde{p}\left(0, s_{n}(j), x, v\right)\left(H-K_{n}\right)\left(s_{n}(j), s_{n}(k), v, y\right) d v \\
& \leq C^{2} \Delta_{\max }^{1 / 2} \sum_{j=0}^{k-1} \Delta_{n}(j+1)\left(s_{n}(k)-s_{n}(j)\right)^{-1 / 2} \zeta^{2,0, k}(y-x) \\
& \leq C^{2} \Delta_{\max }^{1 / 2} s_{n}^{1 / 2}(k) B(1,1 / 2) \zeta^{2,0, k}(y-x) .
\end{aligned}
$$

Now

$$
\begin{aligned}
\tilde{p} \otimes_{n}\left(H+M_{n}\right) \otimes_{n}\left(H+M_{n}\right)\left(0, s_{n}(k), x, y\right) \\
\quad-\tilde{p} \otimes_{n}\left(K_{n}+M_{n}\right) \otimes_{n}\left(K_{n}+M_{n}\right)\left(0, s_{n}(k), x, y\right) \\
=\left(\tilde{p} \otimes_{n} H-\tilde{p} \otimes_{n} K_{n}\right) \otimes_{n}\left(K_{n}+M_{n}\right)\left(0, s_{n}(k), x, y\right) \\
\quad+\tilde{p} \otimes_{n}\left(H+M_{n}\right) \otimes_{n}\left(H-K_{n}\right)\left(0, s_{n}(k), x, y\right) \\
=I+I I .
\end{aligned}
$$

From (5.82) and (5.47) we get

$$
\begin{aligned}
|I| & \leq C^{3} \Delta_{\max }^{1 / 2} B(1,1 / 2) \sum_{j=0}^{k-1} \Delta_{n}(j+1) s_{n}^{1 / 2}(j)\left(s_{n}(k)-s_{n}(j)\right)^{-1 / 2} \zeta^{3,0, k}(y-x) \\
& \leq C^{3} \Delta_{\max }^{1 / 2} B(1,1 / 2) B(3 / 2,1 / 2) s_{n}(k) \zeta^{3,0, k}(y-x) .
\end{aligned}
$$

Proceeding as in the proof of Lemma 5.10 and using Lemma 5.3 instead of Lemma 5.9 we have analogously to $(5.55)$

$$
|I I| \leq C^{3} \Delta_{\max }^{1 / 2} \Gamma^{2}(1 / 2) s_{n}(k) \zeta^{3,0, k}(y-x) .
$$

From $(5.83),(5.84)$ and $(5.85)$ we get

$$
\begin{aligned}
\mid \tilde{p} \otimes_{n}\left(H+M_{n}\right) \otimes_{n}\left(H+M_{n}\right)\left(0, s_{n}(k), x, y\right) \\
\quad-\tilde{p} \otimes_{n}\left(K_{n}+M_{n}\right) \otimes_{n}\left(K_{n}+M_{n}\right)\left(0, s_{n}(k), x, y\right) \mid \\
\leq(2 C)^{3} \Delta_{\max }^{1 / 2} B(1,1 / 2) B(3 / 2,1 / 2) s_{n}(k) \zeta^{3,0, k}(y-x) .
\end{aligned}
$$

Iterative application of analogous arguments gives

$$
\begin{aligned}
& \tilde{p} \otimes_{n}\left(H+M_{n}\right)^{(r)}\left(0, s_{n}(k), x, y\right) \\
& -\tilde{p} \otimes_{n}\left(K_{n}+M_{n}\right)^{(r)}\left(0, s_{n}(k), x, y\right) \\
= & \left(\tilde{p} \otimes_{n}\left(H+M_{n}\right)^{(r-1)}-\tilde{p} \otimes_{n}\left(K_{n}+M_{n}\right)^{(r-1)}\right) \otimes_{n}\left(K_{n}+M_{n}\right)\left(0, s_{n}(k), x, y\right) \\
& +\tilde{p} \otimes_{n}\left(H+M_{n}\right)^{(r-1)} \otimes_{n}\left(H-K_{n}\right)\left(0, s_{n}(k), x, y\right),
\end{aligned}
$$

where

$$
\begin{aligned}
& \left|\tilde{p} \otimes_{n}\left(H+M_{n}\right)^{(r-1)} \otimes_{n}\left(H-K_{n}\right)\left(0, s_{n}(k), x, y\right)\right| \leq \\
& \quad \leq 2 C^{r+2} \Delta_{\max }^{1 / 2} \Gamma^{r+1}(1 / 2) s_{n}^{(r+1) / 2}(k) \zeta^{r+2,0, k}(y-x) / \Gamma((r+2) / 2)
\end{aligned}
$$


and

$$
\begin{aligned}
& \left|\left(\tilde{p} \otimes_{n}\left(H+M_{n}\right)^{(r-1)}-\tilde{p} \otimes_{n}\left(K_{n}+M_{n}\right)^{(r-1)}\right) \otimes_{n}\left(M_{n}+K_{n}\right)\left(0, s_{n}(k), x, y\right)\right| \\
& \quad \leq 2^{r} C^{r+1} \Delta_{\max }^{1 / 2} B(1,1 / 2) \ldots B((r+1) / 2,1 / 2) s_{n}^{r / 2}(k) \zeta^{r+1,0, k}(y-x) .
\end{aligned}
$$

Claim (5.67) follows from (5.87) - (5.89).

Proof of (5.67) for $k=4$. We have

$$
T_{4}=\left|\sum_{r=0}^{n}\left(\tilde{p}-\tilde{p}_{n}\right) \otimes_{n}\left(M_{n}+K_{n}\right)^{(r)}(0,1, x, y)\right|
$$

with

$$
\tilde{p}\left(0, s_{n}(i), x, v\right)=\operatorname{det} \Sigma_{i}^{-1 / 2} \varphi\left(\Sigma_{i}^{-1 / 2}\left(v-x-m_{i}\right)\right),
$$

where $\varphi$ is a standard normal density and where

$$
\Sigma_{i}=\int_{0}^{s_{n}(i)} \Sigma(\tau, v) d \tau, \quad m_{i}=\int_{0}^{s_{n}(i)} m(\tau, v) d \tau .
$$

In notations of Lemma 5.7 we can write $\tilde{p}_{n}\left(0, s_{n}(i), x, v\right)$ in the form

$$
\tilde{p}_{n}\left(0, s_{n}(i), x, v\right)=\operatorname{det} V_{0, i}^{-1 / 2} f_{n}\left(V_{0, i}^{-1 / 2}\left(v-x-\mu_{0, i}\right)\right) .
$$

Note that $V_{0, i}$ and $\mu_{0 . i}$ are integral sums for $\Sigma_{i}$ and $m_{i}$, respectively. By (A5) we easily get

$$
\left\|\mu_{0, i}-m_{i}\right\| \leq C \Delta_{\max ,}\left\|V_{0, i}-\Sigma_{i}\right\| \leq C \Delta_{\max } .
$$

We introduce also

$$
\hat{p}_{n}\left(0, s_{n}(i), x, v\right)=\operatorname{det} V_{0, i}^{-1 / 2} \varphi\left(V_{0, i}^{-1 / 2}\left(v-x-\mu_{0, i}\right)\right) .
$$

Note that $\left|\tilde{p}-\tilde{p}_{n}\right| \leq\left|\tilde{p}-\hat{p}_{n}\right|+\left|\hat{p}_{n}-\tilde{p}_{n}\right|=I+I I$. We estimate first the second term II.

It follows from the proof of Lemma 5.7 (see (5.33)) and from Condition (A4) that

$$
\left|\hat{p}_{n}-\tilde{p}_{n}\right| \leq C i^{-1 / 2} \zeta_{\rho}(v-x) \leq C \Delta_{\max }^{1 / 2} s_{n}^{-1 / 2}(i) \zeta_{\rho}(v-x)
$$

Mimicking the proof of Lemma 5.10 with (5.92) instead of Lemma 5.7 we get

$$
\begin{aligned}
& \left|\left(\hat{p}_{n}-\tilde{p}_{n}\right) \otimes_{n}\left(M_{n}+K_{n}\right)^{(r)}(0,1, x, y)\right| \\
& \quad \leq C^{r+1} \Delta_{\max }^{1 / 2} B(1 / 2,1 / 2) B(1,1 / 2) \ldots B(r / 2,1 / 2) \zeta^{r+1,0, n}(y-x)
\end{aligned}
$$

which immediately gives

$$
\left|\sum_{r=0}^{\infty}\left(\hat{p}_{n}-\tilde{p}_{n}\right) \otimes_{n}\left(M_{n}+K_{n}\right)^{(r)}(0,1, x, y)\right| \leq C \cdot \Delta_{\text {max }}^{1 / 2}\left[1+\|y-x\|^{2 S^{\prime}-2}\right]^{-1}
$$


Differentiating with respect to covariances and means we also get

$$
\left|\left(\tilde{p}-\hat{p}_{n}\right)\left(0, s_{n}(i), x, v\right)\right| \leq C \cdot \Delta_{\max } \zeta_{\rho}(v-x)
$$

and again as in Lemma 5.10 we have

$$
\left|\sum_{r=0}^{\infty}\left(\tilde{p}-\hat{p}_{n}\right) \otimes_{n}\left(M_{n}+K_{n}\right)^{(r)}(0,1, x, y)\right| \leq C \cdot \Delta_{\max }\left[1+\|y-x\|^{2 S^{\prime}-2}\right]^{-1}
$$

From (5.93) and (5.94) we get claim (5.67) for $k=4$.

\section{References.}

Bhattacharya, R. and Rao, R. (1976). Normal approximations and asymptotic expansions. John Wiley \& Sons, New York.

Dahlhaus, R. (1997). Fitting time series models to nonstationary processes. Ann. Statist. 25, 1-37.

Davydov, Y. A. (1980). On strong convergence of distributions of functionals of random processes. I. Theory Probab. Appl. 25, 772-789.

Davydov, Y. A. (1981). On strong convergence of distributions of functionals of random processes. II. Theory Probab. Appl. 26, 258-278.

Doukhan, P. and Nze, A.(1995). Functional estimation for time series: a general approach. Techn. Report.

Franke J., Kreiss J.- P. and Mammen, E. (1996). Bootstrap of kernel smoothing in nonlinear time series. Manuscript.

Gel'fand, I.M. and Shilov, G.E. (1958). Generalized functions, vol.2. Fizmatgiz, Moscow (in russian)

Il'in, A. M., Kalashinikov, A. S. and Oleinik, O. A. (1962). Linear second order parabolic equations. Uspechi Mat. Nauk XVII, 3, 3-146. (in russian)

Kasymdzganova, M. (1981). Local limit theorems for homogenieus Markov chains. Ph.D. Thesis. Moscow Statw Univ. (in russian)

Kloeden, P. E. and Platen, E. (1992). Numerical solution of stochastic differential equations. Springer, Berlin, Heidelberg. 
Konakov, V. D. and Molchanov, S. A. (1984). On the convergence of Markov chains to diffusion processes. Teor. Veroyatn. Mat. Stat.31, 51-64, (in russian) [English translation in Theory Probab. Math. Stat. (1985) 31, 59-73].

Konovalov, A. G. (1981). A local limit theorem on convergence to a diffusion process. Theory Probab. Appl., 26, 581-587.

Kutoyants, Y. A. (1997). Efficiency of the empirical distribution for ergodic diffusion. Bernoulli 3, 445-456.

Kutoyants, Y. A. (1998). Some problems of nonparametric estimation by observations of ergodic diffusion process. Statist. Probab. Letters, to appear.

Kuznetsov, S. E. (1998). Polar boundary sets for superdiffusions and removable lateral singularities for nonlinear parabolic PDEs. Comm. Pure Appl. Math. 51, 303-340.

Masry, E. and Tjøstheim, D. (1994). Nonparametric Estimation and Identification of Nonlinear ARCH Time Series: Strong Convergence and Asymptotic Normality. Econometric Theory 11, 258-289.

McKean, H. P., Singer, I. M. (1967). Curvature and the eigenvalues of the Laplacian. J. Differential Geometry 1, 43-69.

Pinsky, M. A. (1991). Lectures on random evolution. World Scientific, Singapore, New Jersey.

Priestley, M.B. (1988). Nonlinear and Nonstationary Time Series Analysis. Academic Press, London.

Robinson, P. M. (1983). Nonparametric estimators for time series. J. Time Ser. Anal. 4, 185-207.

Skorohod, A. V. (1987). Asymptotic methods for Stochastic differential equations. (in russian). Kiev Naukova dumka.

Thomée, V. (1990). Finite difference methods for linear parabolic equations. In: Handbook of Numerical Analysis Vol. I, (edit. by Ciarlet, P. G. and Lions, J. L.), North Holland, Amsterdam.

Tjøstheim, D. (1994). Non-linear time series: a selective review. Scand. J. Statist. 21, $97-130$.

Tjøstheim, D. and Auestad, B.H. (1994). Nonparametric identification of nonlinear time series: projections. J. Amer. Statist. Assoc. 89, 1398-1409. 